\title{
CATEGORICAL MORITA EQUIVALENCE FOR GROUP-THEORETICAL CATEGORIES
}

\author{
DEEPAK NAIDU
}

\begin{abstract}
A finite tensor category is called pointed if all its simple objects are invertible. We find necessary and sufficient conditions for two pointed semisimple categories to be dual to each other with respect to a module category. Whenever the dual of a pointed semisimple category with respect to a module category is pointed, we give explicit formulas for the Grothendieck ring and for the associator of the dual. This leads to the definition of categorical Morita equivalence on the set of all finite groups and on the set of all pairs $(G, \omega)$, where $G$ is a finite group and $\omega \in H^{3}\left(G, k^{\times}\right)$. A group-theoretical and cohomological interpretation of this relation is given. A series of concrete examples of pairs of groups that are categorically Morita equivalent but have non-isomorphic Grothendieck rings are given. In particular, the representation categories of the Drinfeld doubles of the groups in each example are equivalent as braided tensor categories and hence these groups define the same modular data.
\end{abstract}

\section{INTRODUCTION}

Throughout this paper we work over an algebraically closed field $k$ of characteristic 0 . As pointed out to us by the referee, most results can be extended to the positive characteristic setting using the theory of finite tensor categories. A right module category over a tensor category $\mathcal{C}$ is a semisimple abelian category $\mathcal{M}$ together with a functor $\mathcal{M} \times \mathcal{C} \rightarrow \mathcal{M}$ and certain associativity and unit constraints satisfying some natural axioms (see Ostrik, 2003 (1) and references therein). The dual of a tensor category $\mathcal{C}$ with respect to an indecomposable right module category is the category $\mathcal{C}_{\mathcal{M}}^{*}:=F u n_{\mathcal{C}}(\mathcal{M}, \mathcal{M})$ whose objects are $\mathcal{C}$-module functors from $\mathcal{M}$ to itself and morphisms are natural module transformations. The category $\mathcal{C}_{\mathcal{M}}^{*}$ is a tensor category with tensor product being composition of module functors. Moreover, $\mathcal{M}$ becomes a left module category over $\mathcal{C}_{\mathcal{M}}^{*}$ in an obvious way. The duality of tensor categories is known to be an equivalence relation Müger, 2003. A fusion category over $k$ is a $k$-linear semisimple rigid tensor category with finitely many isomorphism classes of simple objects and finite-dimensional Hom-spaces such that the neutral object in simple (see Etingof, Nikshych and Ostrik, 2005).

A finite tensor category is said to be pointed if all its simple object are invertible. Every pointed semisimple category is equivalent to the fusion category $\operatorname{Vec}(G, \omega)$ whose objects are vector spaces graded by the finite group $G$ and whose associativity constraint is given by the 3 -cocycle $\omega \in Z^{3}\left(G, k^{\times}\right)$. Let us denote $\operatorname{Vec}(G):=\operatorname{Vec}(G, 1)$. A fusion category is called group-theoretical if it is equivalent to the dual of $\operatorname{Vec}(G, \omega)$ with respect to some indecomposable right module category for some finite group $G$ and 3-cocycle $\omega \in Z^{3}\left(G, k^{\times}\right)$.

In this paper we use the notion of weak Morita equivalence Müger, 2003 of tensor categories to define and study an equivalence relation called categorical Morita equivalence on the set of all finite groups and on the set of all pairs $(G, \omega)$, where $G$ is a finite group and $\omega \in H^{3}\left(G, k^{\times}\right)$. Namely, we say that two groups $G$ and $G^{\prime}$ (respectively, two pairs 
$(G, \omega)$ and $\left.\left(G^{\prime}, \omega^{\prime}\right)\right)$ are categorically Morita equivalent if $\operatorname{Vec}(G)$ is dual to $V e c\left(G^{\prime}\right)$ (respectively, $\operatorname{Vec}(G, \omega)$ is dual to $\left.\operatorname{Vec}\left(G^{\prime}, \omega^{\prime}\right)\right)$ with respect to some indecomposable right module category. This equivalence relation extends the notion of isocategorical groups, i.e., groups with equivalent tensor categories of representations, studied in Davydov, 2001 and Etingof and Gelaki, 2001. Our motivation to study categorical Morita equivalence of finite groups comes from the question about existence of semisimple Hopf algebras with non grouptheoretical representation categories asked in Etingof, Nikshych and Ostrik, 2005, Question 8.45]. We think that understanding equivalence classes of categorically Morita equivalent groups is a natural step towards answering this question.

The main results of this paper are: (1) Computation of the dual of $\operatorname{Vec}(G, \omega)$ with respect to an indecomposable module category when the dual is pointed, including explicit formulas for the Grothendieck ring and the associated 3-cocycle. (2) Necessary and sufficient conditions for two pointed semisimple categories to be dual to each other with respect to a module category. (3) A series of concrete examples of pairs of groups $\left(G_{1}, G_{2}\right)$ that are categorically Morita equivalent but have non-isomorphic Grothendieck rings (and hence, inequivalent representation categories). A consequence of the categorical Morita equivalence of these groups is that the representation categories of their Drinfeld doubles $\operatorname{Rep}\left(\mathrm{D}\left(G_{1}\right)\right)$ and $\operatorname{Rep}\left(\mathrm{D}\left(G_{2}\right)\right)$ are equivalent as braided tensor categories and so in particular these groups define the same modular data. To the best of our knowledge these are first examples of finite groups with this property, cf. a discussion of a finite group modular data in Coste, Gannon, and Ruelle, 2000. These results are contained in Theorems 4.5, 4.9, 5.8 and Corollary 6.2 .

The paper is organized as follows: in Section 2 we recall necessary definition and facts from homological algebra. We also recall the notions of module categories and duals of tensor categories. In Section 3 we give necessary and sufficient conditions for the dual of $V e c(G, \omega)$ with respect to an indecomposable right module category to be pointed. In Section 4 we show that the Grothendieck ring of the dual of $\operatorname{Vec}(G, \omega)$ with respect to an indecomposable right module category when the dual is pointed is the group ring of a certain crossed product of groups. We also find an explicit formula for the 3-cocycle associated to the dual category. In Section 5 we introduce the categorical Morita equivalence on the set of all finite groups and on the set of all pairs $(G, \omega)$, where $G$ is a finite group and $\omega \in H^{3}\left(G, k^{\times}\right)$. We give a grouptheoretical and cohomological interpretation of these relations. In the final section, Section 6 , we give a series of examples of pairs of groups that are categorically Morita equivalent but have non-isomorphic Grothendieck rings.

All categories considered in this paper are assumed to abelian, semisimple and $k$-linear with finite dimensional Hom-spaces. We will also assume that the number of isomorphism classes of simple objects in any category is finite. All functors are assumed to be additive and $k$-linear.

\section{Preliminaries}

2.1. Cohomology of groups and Shapiro's lemma. Let $G$ be a finite group and $M$ be a left $G$-module with action denoted by $(g, m) \mapsto g \triangleright m$, for $g \in G, m \in M$. We define a cochain complex $C(G, M)=\left(C^{n}(G, M)\right)_{n \geq 0}$ of $G$ with coefficients in $M$ as follows. Let $G^{n}=G \times \cdots \times G$ ( $n$ factors) and $C^{n}(G, M)=F u n\left(G^{n}, M\right)$ be the set of all $n$-cochains. By convention, $G^{0}(G, M)=M$. A $n$-cochain $f$ is said to be normalized if $f\left(g_{1}, g_{2}, \ldots, g_{n}\right)=0_{M}$ whenever $g_{i}=1_{G}$ for some $i \in\{1,2, \ldots, n\}$. All $n$-cochains are assumed to be normalized. 
Let $\delta^{n}: C^{n}(G, M) \rightarrow C^{n+1}(G, M)$ be the coboundary operator given by

$$
\begin{aligned}
\left(\delta^{n} f\right)\left(g_{1}, \ldots, g_{n+1}\right)= & g_{1} \triangleright f\left(g_{2}, \ldots, g_{n+1}\right) \\
& +\sum_{i=1}^{n}(-1)^{i} f\left(g_{1}, \ldots, g_{i-1}, g_{i} g_{i+1}, \ldots, g_{n+1}\right) \\
& +(-1)^{n+1} f\left(g_{1}, \ldots, g_{n}\right)
\end{aligned}
$$

for all $f \in C^{n}(G, M)$.

If $M$ is a right $G$-module, we denote the action by $(m, g) \mapsto m \triangleleft g$, for $g \in G, m \in M$. Also, define $\underline{\delta}^{n}: C^{n}(G, M) \rightarrow C^{n+1}(G, M)$ by

$$
\begin{aligned}
\left(\underline{\delta}^{n} f\right)\left(g_{1}, \ldots, g_{n+1}\right)= & f\left(g_{2}, \ldots, g_{n+1}\right) \\
& +\sum_{i=1}^{n}(-1)^{i} f\left(g_{1}, \ldots, g_{i-1}, g_{i} g_{i+1}, \ldots, g_{n+1}\right) \\
& +(-1)^{n+1}\left(f\left(g_{1}, \ldots, g_{n}\right) \triangleleft g_{n+1}\right)
\end{aligned}
$$

for all $f \in C^{n}(G, M)$.

Let $Z^{n}(G, M)=\operatorname{Ker}\left(\delta^{n}\right)$ be the set of $n$-cocycles and $B^{n}(G, M)=\operatorname{Im}\left(\delta^{n-1}\right)$ be the space of $n$-coboundaries. Let $\underline{Z}^{n}(G, M)=\operatorname{Ker}\left(\underline{\delta}^{n}\right)$ and $\underline{B}^{n}(G, M)=\operatorname{Im}\left(\underline{\delta}^{n-1}\right)$. The $n$-th cohomology group $H^{n}(G, M)$ of $G$ with coefficients in $M$ is the quotient $Z^{n}(G, M) / B^{n}(G, M)$ $(n \geq 1)$. Also, let $\underline{H}^{n}(G, M)=\underline{Z}^{n}(G, M) / \underline{B}^{n}(G, M)$. When we write an element of the cohomology groups as $\bar{\omega}$, we will mean by this the class represented by the cocycle $\omega$.

Any homomorphism $a: G^{\prime} \rightarrow G$ between the groups $G$ and $G^{\prime}$ induces a map between their cohomology groups:

$$
H^{n}(G, M) \rightarrow H^{n}\left(G^{\prime}, M\right):(a, \bar{\omega}) \mapsto \omega^{a}:=\omega \circ a^{\times n},
$$

Let $H$ be a subgroup of $G$. Let $p: G \rightarrow H \backslash G$ be the usual surjection, i.e., $p(g):=H g$, for all $g \in G$. Throughout this paper we will denote $p\left(1_{G}\right)$ by 1 . For each $x \in H \backslash G$ choose a representative $u(x)$ in $\mathrm{G}$; i.e., an element $u(x)$ with $p u(x)=x$. In particular, choose $u(1)=1_{G}$. The set $H \backslash G$ is a right $G$-set with the obvious action: $x \triangleleft g:=p(u(x) g)$, for $x \in H \backslash G$ and $g \in G$. Also, the set $\{u(x) \mid x \in H \backslash G\}$ is a right $G$-set: $u(x) \triangleleft g=u(x \triangleleft g)$, for $x \in H \backslash G$ and $g \in G$. The elements $u(x) g$ and $u(x \triangleleft g)$ differ by an element $\kappa_{x, g}$ of $H$, for $x \in H \backslash G$ and $g \in G$ :

$$
u(x) g=\kappa_{x, g} u(x \triangleleft g)
$$

The following relation holds:

$$
\kappa_{x, g_{1} g_{2}}=\kappa_{x, g_{1}} \kappa_{x \triangleleft g_{1}, g_{2}}
$$

for any $x \in H \backslash G$ and $g_{1}, g_{2} \in G$

The set $\operatorname{Fun}\left(H \backslash G, k^{\times}\right)$of functions from $H \backslash G$ to $k^{\times}$is a left $G$-module: $(g \triangleright f)(x)=$ $f(x \triangleleft g)$, for $x \in H \backslash G$ and $g \in G$. Let us regard $k^{\times}$as a trivial left $H$-module. It is easy to see that $\operatorname{Fun}\left(H \backslash G, k^{\times}\right)$is isomorphic to the coinduced module $\operatorname{Coind}_{H}^{G}\left(k^{\times}\right)=\operatorname{Hom}_{H}\left(G, k^{\times}\right)$. From now on we will identify the coinduced module $\operatorname{Coind}_{H}^{G}\left(k^{\times}\right)$with $\operatorname{Fun}\left(H \backslash G, k^{\times}\right)$.

Let $C:=\operatorname{Coind}_{H}^{G}\left(k^{\times}\right)$and $K:=H \backslash G$. The action of $G$ on $K$ restricts to an action of $H$ on $K$. Let $K^{H}$ denote the set of elements of $K$ that are stable under the action of $H$. Note 
that $K^{H}$ forms a group that is isomorphic to $H \backslash N_{G}(H)$, where $N_{G}(H)$ is the normalizer of $H$ in $G$. By $\widehat{H}$, we will mean the group $\operatorname{Hom}\left(H, k^{\times}\right)$of 1-dimensional representations of $H$.

By Shapiro's Lemma there is an isomorphism between $H^{n}(G, C)$ and $H^{n}\left(H, k^{\times}\right)$for each $n \in \mathbb{N}$. It is well known that the restriction maps induces this isomorphism. We will need the explicit form of the inverse of the restriction map when $n=1,2$. Lemmas 2.1 and 2.2 provide this.

Lemma 2.1. The following map induces an isomorphism between $H^{1}\left(H, k^{\times}\right)=\widehat{H}$ and $H^{1}(G, C)$ :

$$
\varphi_{1}: Z^{1}\left(H, k^{\times}\right) \rightarrow Z^{1}(G, C), \quad\left(\varphi_{1}(\rho)(g)\right)(x)=\rho\left(\kappa_{x, g}\right)
$$

for any $\rho \in Z^{1}\left(H, k^{\times}\right), g \in G, x \in K$.

Proof. We will first show that $\varphi_{1}(\rho) \in Z^{1}(G, C)$ for any $\rho \in Z^{1}\left(H, C^{\times}\right)$. We need to show that $\varphi_{1}(\rho)$ satisfies the equation:

$$
\begin{gathered}
\left(\varphi_{1}(\rho)\left(g_{1}\right)\right)(x)\left(\varphi_{1}(\rho)\left(g_{2}\right)\right)\left(x \triangleleft g_{1}\right)=\left(\varphi_{1}(\rho)\left(g_{1} g_{2}\right)\right)(x) \\
\Leftrightarrow \rho\left(\kappa_{x, g_{1}}\right) \rho\left(\kappa_{x \triangleleft g_{1}, g_{2}}\right)=\rho\left(\kappa_{x, g_{1} g_{2}}\right)
\end{gathered}
$$

The 1-cocycle condition on $\rho$ is:

$$
\rho\left(h_{1}\right) \rho\left(h_{2}\right)=\rho\left(h_{1} h_{2}\right)
$$

Put $h_{1}=\kappa_{x g_{1}}$ and $h_{2}=\kappa_{x \triangleleft g_{1}, g_{2}}$ in the above equation and use Equation (3) to obtain the desired equation.

The map $\varphi_{1}$ induces a map:

$$
\widetilde{\varphi_{1}}: H^{1}\left(H, k^{\times}\right) \rightarrow H^{1}(G, C)
$$

One can show that the map $\widetilde{\varphi_{1}}$ does not depend on the choice of the function $u: K \rightarrow G$. Let $\psi_{1}$ denote the restriction map.

$$
\psi_{1}: Z^{1}(G, C) \rightarrow Z^{1}\left(H, k^{\times}\right), \quad \psi_{1}(\gamma)(h)=\gamma(h)(1)
$$

for any $\gamma \in Z^{1}(G, C)$ and $h \in H$. Let $\widetilde{\psi_{1}}$ denote the induced map:

$$
\widetilde{\psi_{1}}: H^{1}(G, C) \rightarrow H^{1}\left(H, k^{\times}\right)
$$

It remains to show that the maps $\widetilde{\varphi_{1}}$ and $\widetilde{\psi_{1}}$ are inverse to each other. It suffice to show that $\widetilde{\psi_{1}} \circ \widetilde{\varphi_{1}}=I d_{H^{1}\left(H, C^{\times}\right)}$. Actually, we show that $\psi_{1} \circ \varphi_{1}=I d_{Z^{1}\left(H, k^{\times}\right)}$. Pick any $\rho \in Z^{1}\left(H, k^{\times}\right)$. Then $\psi_{1}\left(\varphi_{1}(\rho)\right)(h)=\left(\varphi_{1}(\rho)(h)\right)(1)=\rho\left(\kappa_{1, h}\right)=\rho(h)$ for all $h \in H$ and the lemma is proved.

Lemma 2.2. The following map induces an isomorphism between $H^{2}\left(H, k^{\times}\right)$and $H^{2}(G, C)$ :

$$
\varphi: Z^{2}\left(H, k^{\times}\right) \rightarrow Z^{2}(G, C), \quad\left(\varphi(\mu)\left(g_{1}, g_{2}\right)\right)(x)=\mu\left(\kappa_{x, g_{1}}, \kappa_{x \triangleleft g_{1}, g_{2}}\right)
$$

for any $\mu \in Z^{2}\left(H, k^{\times}\right), g_{1}, g_{2} \in G, x \in K$. 
Proof. We will first show that $\varphi(\mu) \in Z^{2}(G, C)$ for any $\mu \in Z^{2}\left(H, k^{\times}\right)$. We need to show that $\varphi(\mu)$ satisfies the following equation for all $g_{1}, g_{2}, g_{3} \in G$ and $x \in K$.

$$
\begin{aligned}
& \left(\varphi(\mu)\left(g_{2}, g_{3}\right)\right)\left(x \triangleleft g_{1}\right)\left(\varphi(\mu)\left(g_{1}, g_{2} g_{3}\right)\right)(x)=\left(\varphi(\mu)\left(g_{1} g_{2}, g_{3}\right)\right)(x)\left(\varphi(\mu)\left(g_{1}, g_{2}\right)\right)(x) \\
\Leftrightarrow & \mu\left(\kappa_{x \triangleleft g_{1}, g_{2}}, \kappa_{x \triangleleft g_{1} g_{2}, g_{3}}\right) \mu\left(\kappa_{x, g_{1}}, \kappa_{x \triangleleft g_{1}, g_{2} g_{3}}\right)=\mu\left(\kappa_{x, g_{1} g_{2}}, \kappa_{x \triangleleft g_{1} g_{2}, g_{3}}\right) \mu\left(\kappa_{x, g_{1}}, \kappa_{x \triangleleft g_{1}, g_{2}}\right)
\end{aligned}
$$

The 2-cocycle condition on $\mu$ is:

$$
\mu\left(h_{2}, h_{3}\right) \mu\left(h_{1}, h_{2} h_{3}\right)=\mu\left(h_{1} h_{2}, h_{3}\right) \mu\left(h_{1}, h_{2}\right)
$$

for any $h_{1}, h_{2}, h_{3} \in H$. Put $h_{1}=\kappa_{x, g_{1}}, h_{2}=\kappa_{x \triangleleft g_{1}, g_{2}}$ and $h_{3}=\kappa_{x \triangleleft g_{1} g_{2}, g_{3}}$ in the above equation and use equation (3) to obtain the desired equation.

One can show that $\varphi$ preserves coboundaries, hence it induces a map:

$$
\widetilde{\varphi}: H^{2}\left(H, k^{\times}\right) \rightarrow H^{2}(G, C)
$$

Let $\psi$ denote the restriction map:

$$
\psi: Z^{2}(G, C) \rightarrow Z^{2}\left(H, k^{\times}\right), \quad \psi(\gamma)\left(h_{1}, h_{2}\right)=\gamma\left(h_{1}, h_{2}\right)(1)
$$

for any $\gamma \in Z^{2}(G, C)$ and $h_{1}, h_{2} \in H$. Let $\widetilde{\psi}$ denote the induced map:

$$
\widetilde{\psi}: H^{2}(G, C) \rightarrow H^{2}\left(H, k^{\times}\right)
$$

It remains to show that the maps $\widetilde{\varphi}$ and $\widetilde{\psi}$ are inverse to each other. It suffice to show that $\widetilde{\psi} \circ \widetilde{\varphi}=I d_{H^{2}\left(H, k^{\times}\right)}$. Actually, we show that $\psi \circ \varphi=I d_{Z^{2}\left(H, k^{\times}\right)}$. Pick any $\mu \in Z^{2}\left(H, k^{\times}\right)$. Then $\psi(\varphi(\mu))\left(h_{1}, h_{2}\right)=\left(\varphi(\mu)\left(h_{1}, h_{2}\right)\right)(1)=\mu\left(\kappa_{1, h_{1}}, \kappa_{1 \triangleleft h_{1}, h_{2}}\right)=\mu\left(h_{1}, h_{2}\right)$ for all $h_{1}, h_{2} \in H$ and the lemma is proved.

There is a right action of $K^{H}$ on $C^{n}(G, C)$ :

$$
(\gamma, x) \mapsto{ }^{x} \gamma, \quad{ }^{x} \gamma\left(g_{1}, \ldots, g_{n}\right)(y):=\gamma\left(g_{1}, \ldots, g_{n}\right)(p(u(x) u(y))
$$

for all $\gamma \in C^{n}(G, C), g_{1}, \ldots, g_{n} \in G, x \in K^{H}$, and $y \in K$

It is routine to check that the above action is independent of the function $u$. This induces a right action of $K^{H}$ on $Z^{n}(G, C)$ and $H^{n}(G, C)$. If $H$ is normal in $G$, then $K^{H}=K$ and

$$
{ }^{x} \gamma\left(g_{1}, \ldots, g_{n}\right)(y):=\gamma\left(g_{1}, \ldots, g_{n}\right)(x y)
$$

for all $\gamma \in C^{n}(G, C), g_{1}, \ldots, g_{n} \in G, x, y \in K$.

Also, if $H$ is normal in $G$, then $Z^{n}\left(H, k^{\times}\right)$is a right $G$-module:

$$
(\mu, g) \mapsto \mu^{g}, \quad \mu^{g}\left(h_{1}, \ldots, h_{n}\right)=\mu\left(g h_{1} g^{-1}, \ldots, g h_{n} g^{-1}\right)
$$

for all $\mu \in Z^{n}\left(H, k^{\times}\right), g \in G$ and $h_{1}, \ldots, h_{n} \in H$. 
If $H$ is abelian and normal in $G$, then $Z^{n}\left(H, k^{\times}\right)$becomes a right $K$-module:

$$
(\mu, x) \mapsto \mu^{u(x)}
$$

for all $\mu \in Z^{n}\left(H, k^{\times}\right)$and $x \in K$.

The above induces an action of $K$ on $H^{n}\left(H, k^{\times}\right)$.

Lemma 2.3. If $H$ is abelian and normal in $G$, then the map $\psi_{1}$ defined in (5) is a $K$-module map.

Proof. Pick any $\gamma \in Z^{1}(G, C)$ and $y \in K$. We have $\psi_{1}\left({ }^{y} \gamma\right)(h)=\left({ }^{y} \gamma\right)(h)(1)=\gamma(h)(y)$ and $\left(\psi_{1}(\gamma)^{y}\right)(h)=\psi_{1}(\gamma)\left(u(y) h u(y)^{-1}\right)=\gamma\left(u(y) h u(y)^{-1}\right)(1)$. By Lemma 2.1] we know that $\gamma=\left(\delta^{1} \alpha\right) \varphi_{1}(\rho)$ for some $\alpha \in C$ and $\rho \in \widehat{H}$.

We have,

$$
\begin{aligned}
\gamma(h)(y) & =\left(\left(\delta^{1} \alpha\right) \varphi_{1}(\rho)\right)(h)(y) \\
& =\frac{\alpha(y \triangleleft h)}{\alpha(y)} \rho\left(\kappa_{y, h}\right) \\
& =\rho\left(u(y) h u(y)^{-1}\right)
\end{aligned}
$$

and

$$
\begin{aligned}
\gamma\left(u(y) h u(y)^{-1}\right)(1) & =\left(\left(\delta^{1} \alpha\right) \varphi_{1}(\rho)\right)\left(u(y) h u(y)^{-1}\right)(1) \\
& =\frac{\alpha\left(1 \triangleleft u(y) h u(y)^{-1}\right)}{\alpha(1)} \rho\left(\kappa_{\left.1, u(y) h u(y)^{-1}\right)}\right. \\
& =\rho\left(u(y) h u(y)^{-1}\right) .
\end{aligned}
$$

Lemma 2.4. If $H$ is abelian and normal in $G$, then the map $\widetilde{\varphi}$ defined in (8) is a $K$-module map.

Proof. Pick any $\mu \in Z^{2}\left(H, k^{\times}\right)$. In order to show that the map $\widetilde{\varphi}$ is $K$-linear it suffices to show that $\psi\left({ }^{y} \varphi(\mu)\right)$ is cohomologous to $\psi\left(\varphi\left(\mu^{y}\right)\right)=\mu^{y}$ in $H^{2}\left(H, k^{\times}\right)$for all $y \in K$. We will actually show that $\psi\left({ }^{y} \varphi(\mu)\right)=\mu^{y}$. We have,

$$
\begin{aligned}
\psi\left({ }^{y} \varphi(\mu)\right)\left(h_{1}, h_{2}\right) & ={ }^{y} \varphi(\mu)\left(h_{1}, h_{2}\right)(1) \\
& =\varphi(\mu)\left(h_{1}, h_{2}\right)(y) \\
& =\mu\left(\kappa_{y, h_{1}}, \kappa_{y \triangleleft h_{1}, h_{2}}\right) \\
& =\mu\left(\kappa_{y, h_{1}}, \kappa_{y, h_{2}}\right) \\
& =\mu\left(u(y) h_{1} u(y)^{-1}, u(y) h_{2} u(y)^{-1}\right) \\
& =\mu^{y}\left(h_{1}, h_{2}\right)
\end{aligned}
$$

for all $h_{1}, h_{2} \in H$. So $\psi\left({ }^{y} \varphi(\mu)\right)=\mu^{y}$ and the lemma is proved. 
2.2. The fusion category $\operatorname{Vec}(G, \omega)$. We refer the reader to Bakalov and Kirillov Jr., 2001 for definition and basic properties of tensor categories. A category is called skeletal if all isomorphic objects in the category are actually equal. Every category is equivalent to a skeletal category. It is convenient to work with a skeletal category $\mathcal{V}(G, \omega)$ equivalent to $\operatorname{Vec}(G, \omega)$. Let $\mathcal{V}(G, \omega)$ be a semisimple tensor category with simple objects $g, g \in G$. The tensor product is defined by $g_{1} \otimes g_{2}=g_{1} g_{2}$, and the associativity isomorphisms are $\omega\left(g_{1}, g_{2}, g_{3}\right) i d_{g_{1} g_{2} g_{3}}$. The unit object is $1_{G}$. The left and right unit isomorphisms are $\omega\left(1_{G}, 1_{G}, g\right) i d_{g}$ and $\omega\left(g, 1_{G}, 1_{G}\right) i d_{g}$, repectively. The previous statement follows from the triangle axiom for tensor categories. Since we can assume that all cocycles are normalized, the left and right unit isomorphisms are the identity morphisms. The left and right dual objects of $g$ are $g^{*}={ }^{*} g=g^{-1}$. If $G^{\prime}$ is another group and $\omega^{\prime} \in Z^{3}\left(G^{\prime}, k^{\times}\right)$, then $\mathcal{V}(G, \omega) \cong \mathcal{V}\left(G^{\prime}, \omega^{\prime}\right)$ if and only if there is an isomorphism $a: G \rightarrow G^{\prime}$ such that $\omega^{\prime}$ and $\omega^{a}$ are cohomologous.

2.3. Module categories. Recall some definitions from Ostrik, 2003 (1):

Definition 2.5. A right module category over a tensor category $\left(\mathcal{C}, \otimes, 1_{\mathcal{C}}, \alpha, \lambda, \rho\right)$ with unit object $1_{\mathcal{C}}$, associativity constraint $\alpha$, left unit constraint $\lambda$, and right unit constraint $\rho$, is a category $\mathcal{M}$ together with an exact bifunctor $\otimes: \mathcal{M} \times \mathcal{C} \rightarrow \mathcal{M}$ and functorial associativity and unit isomorphisms: $\mu_{M, X, Y}: M \otimes(X \otimes Y) \rightarrow(M \otimes X) \otimes Y, \tau_{M}: M \otimes 1_{\mathcal{C}} \rightarrow M$ for any $X, Y \in \mathcal{C}, M \in \mathcal{M}$ such that the following two diagrams commute.
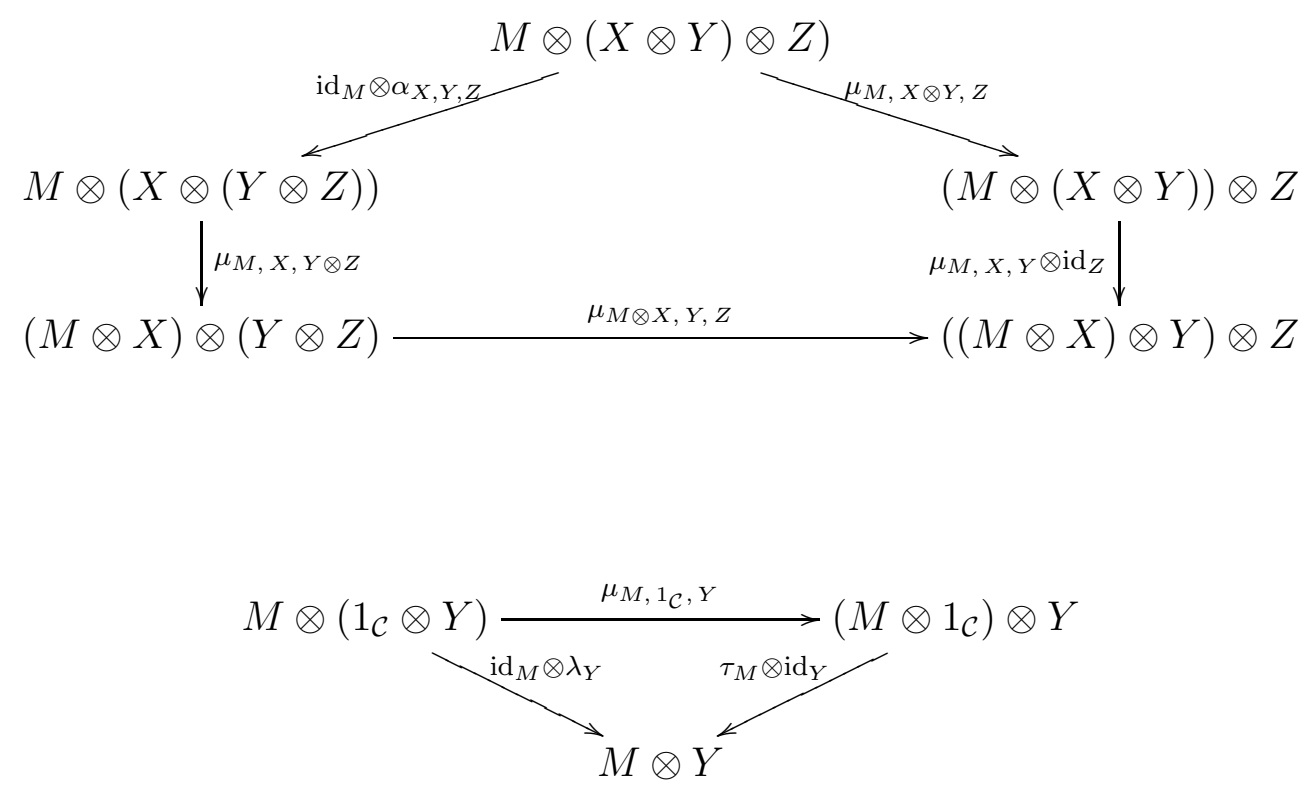

Definition 2.6. Let $\left(\mathcal{M}_{1}, \mu^{1}, \tau^{1}\right)$ and $\left(\mathcal{M}_{2}, \mu^{2}, \tau^{2}\right)$ be two right module categories over a tensor category $\mathcal{C}$. A module functor from $\mathcal{M}_{1}$ to $\mathcal{M}_{2}$ is a functor $F: \mathcal{M}_{1} \rightarrow \mathcal{M}_{2}$ together 
with functorial isomorphisms $\gamma_{M, X}: F(M \otimes X) \rightarrow F(M) \otimes X$ for any $X \in \mathcal{C}, M \in \mathcal{M}_{1}$ such that the following two diagrams commute.
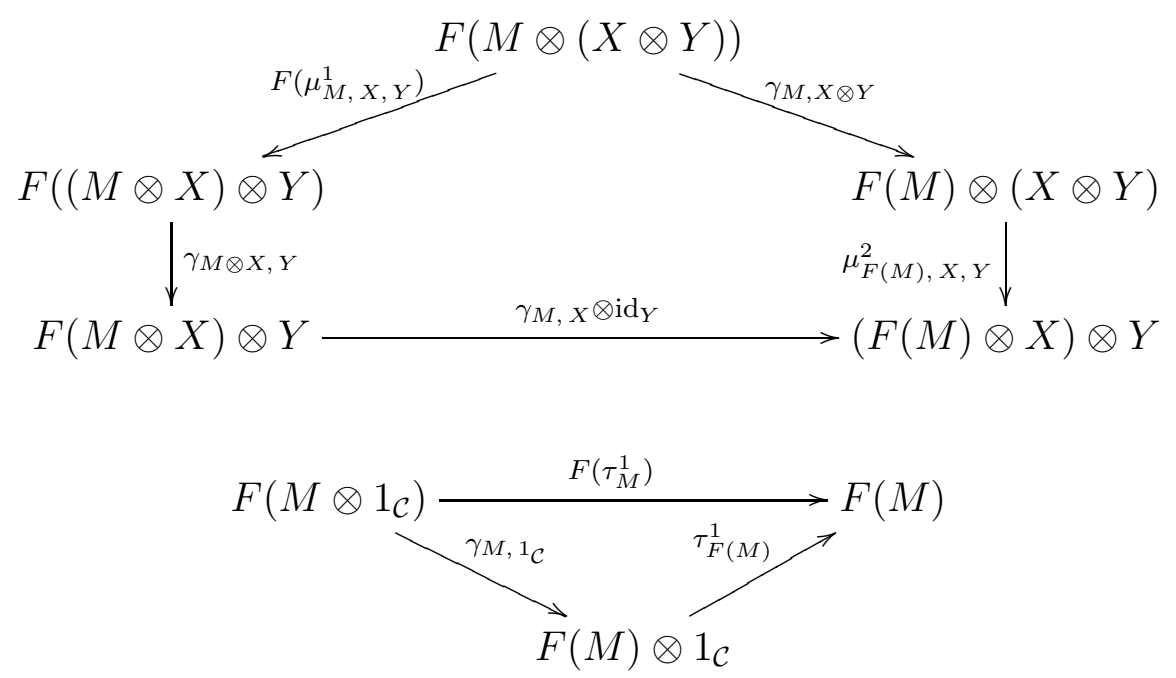

Two module categories $\mathcal{M}_{1}$ and $\mathcal{M}_{2}$ over $\mathcal{C}$ are equivalent if there exists a module functor from $\mathcal{M}_{1}$ to $\mathcal{M}_{2}$ which is an equivalence of categories. For two module categories $\mathcal{M}_{1}$ and $\mathcal{M}_{2}$ over a tensor category $\mathcal{C}$ their direct sum is the category $\mathcal{M}_{1} \oplus \mathcal{M}_{2}$ with the obvious module category structure. A module category is indecomposable if it is not equivalent to a direct sum of two non-trivial module categories.

Definition 2.7. Let $\mathcal{M}_{1}$ and $\mathcal{M}_{2}$ be two right module categories over a tensor category $\mathcal{C}$. Let $\left(F^{1}, \gamma^{1}\right)$ and $\left(F^{2}, \gamma^{2}\right)$ be module functors from $\mathcal{M}_{1}$ to $\mathcal{M}_{2}$. A natural module transformation from $\left(F^{1}, \gamma^{1}\right)$ to $\left(F^{2}, \gamma^{2}\right)$ is a natural transformation $\eta: F^{1} \rightarrow F^{2}$ such that the following square commutes for all $M \in \mathcal{M}, X \in \mathcal{C}$.

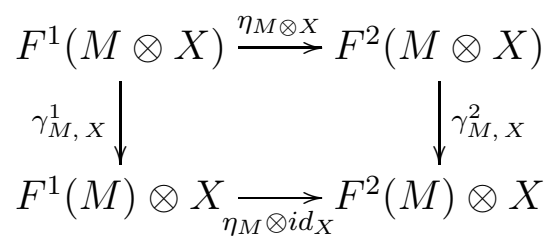

Example 2.8. Let us recall a description of indecomposable module categories over $\mathcal{V}(G, \omega)$ given in Ostrik, 2003 (2) . Let $\mathcal{M}$ be an indecomposable right module category over $\mathcal{V}(G, \omega)$ with module category structure $\mu$. Without loss of generality we may assume that $\mathcal{M}$ is skeletal. The set of simple objects of $\mathcal{M}$ is a transitive right $G$-set and hence can be identified with the set of right cosets $H \backslash G=K$ for some subgroup $H$ of $G$. So the set of all simple objects of $\mathcal{M}, \operatorname{Irr}(\mathcal{M})=K$. All the isomorphisms $\mu_{x, g_{1}, g_{2}}, x \in K, g_{1}, g_{2} \in G$ are given by scalars. So we can regard $\mu$ as an element of $C^{2}(G, C)$ :

$$
\mu\left(g_{1}, g_{2}\right)(x):=\mu_{x, g_{1}, g_{2}}, \quad x \in K, g_{1}, g_{2} \in G
$$

We may assume that the 2-cochain $\mu$ is normalized. Since the unit constraint in $\mathcal{V}(G, \omega)$ is trivial, the commutativiy of triangle (12) implies that the unit constraint in $\mathcal{M}$ is trivial. Let 
us regard $\omega$ as an element of $Z^{3}(G, C) \subset C^{3}(G, C)$ by treating $\omega\left(g_{1}, g_{2}, g_{3}\right)$ as a constant function on $K$, for all $g_{1}, g_{2}, g_{3} \in G$. The commutativity of the pentagon (11) implies that

$$
\delta^{2} \mu=\omega
$$

This in particular means that $\omega$ restricted to $H \times H \times H$ represents the trivial class in $H^{3}\left(H, k^{\times}\right)$. Let $L_{H, \omega}:=\left\{\mu \in C^{2}(G, C) \mid \delta^{2} \mu=\omega\right\}$. Two elements in $L_{H, \omega}$ give rise to equivalent module categories if and only if the differ by some element in $B^{2}(G, C)$. Define an equivalence relation on $L_{H, \omega}$ : two elements in $L_{H, \omega}$ are equivalent if and only if the differ by an element in $B^{2}(G, C)$. We denote the set of equivalence classes of $L_{H, \omega}$ under the previous relation by $\bar{L}_{H, \omega}$. The sets $\bar{L}_{H, \omega}$ and $H^{2}\left(H, k^{\times}\right)$are in bijection.

2.4. The dual category. Let $\mathcal{C}$ be a tensor category and $\mathcal{M}$ be an indecomposable right module category over $\mathcal{C}$.

Definition 2.9. The dual category of $\mathcal{C}$ with respect to $\mathcal{M}$ is the category $\mathcal{C}_{\mathcal{M}}^{*}:=F u n_{\mathcal{C}}(\mathcal{M}, \mathcal{M})$ whose objects are $\mathcal{C}$-module functors from $\mathcal{M}$ to itself and morphisms are natural module transformations.

The category $\mathcal{C}_{\mathcal{M}}^{*}$ is tensor with tensor product being composition of module functors. Let $\left(\gamma^{1}, F^{1}\right),\left(\gamma^{2}, F^{2}\right) \in \operatorname{Obj}\left(\mathcal{C}_{\mathcal{M}}^{*}\right)$, where $\gamma^{1}, \gamma^{2}$ represent the module functor structure on the functors $F^{1}$ and $F^{2}$, respectively. Then, $\left(\gamma^{1}, F^{1}\right) \otimes\left(\gamma^{2}, F^{2}\right)=\left(\gamma, F^{1} \circ F^{2}\right)$, where $\gamma$ is defined as: $\gamma_{M, X}:=\gamma_{F^{2}(M), X}^{1} \circ F^{1}\left(\gamma_{M, X}^{2}\right)$ for any $M \in \mathcal{M}, X \in \mathcal{C}$. Let $\eta:\left(\gamma^{1}, F^{1}\right) \rightarrow\left(\gamma^{2}, F^{2}\right)$ and $\eta^{\prime}:\left(\gamma^{3}, F^{3}\right) \rightarrow\left(\gamma^{4}, F^{4}\right)$ be morphisms in $\mathcal{C}_{\mathcal{M}}^{*}$, i.e., natural module transformations. Then their tensor product $\eta \otimes \eta^{\prime}$ is defined as: $\left(\eta \otimes \eta^{\prime}\right)(M):=\eta_{F^{4}(M)} \circ F^{1}\left(\eta_{M}^{\prime}\right)$.

Remark 2.10. The Frobenius-Perron dimension FPdim $(X)$ of a simple object $X \in \operatorname{Obj}(\mathcal{C})$ is the Frobenius-Perron eigenvalue of the matrix coming from multiplication of the set of isomorphism classes of all simple objects in $\mathcal{C}$ by $X$. The Frobenius-Perron dimension $F P \operatorname{dim}(\mathcal{C})$ of the fusion category $\mathcal{C}$ is the sum of squares of the Frobenius-Perron dimension of the isomorphism classes of simple objects. It is know that if $\mathcal{C}$ is a fusion category and $\mathcal{M}$ is abelian and semisimple then $\mathcal{C}_{\mathcal{M}}^{*}$ is a fusion category. It is also known that $F P \operatorname{dim}(\mathcal{C})=F P \operatorname{dim}\left(\mathcal{C}_{\mathcal{M}}^{*}\right)$. See Etingof, Nikshych and Ostrik, 2005 for a treatment on Frobenius-Perron dimensions.

\section{NECESSARY AND SUfFICIENT CONDItion FOR THE DUAL OF A POINTED CATEGORY TO BE POINTED}

We fix the following notation for this and the next section. Let $K:=H \backslash G$ and $C:=$ $\operatorname{Coind}_{H}^{G} k^{\times}$. Let $u: K \rightarrow G$ be a function satisfying $p \circ u=i d_{K}$ and $u\left(p\left(1_{G}\right)\right)=1_{G}$, where $p: G \rightarrow K$ is the usual surjection. Let $\kappa: K \times G \rightarrow H$ be the function satisfying Equation (2). Let $\mathcal{C}:=\mathcal{V}(G, \omega)$ and let $\mathcal{M}=\mathcal{M}(H, \mu)$ denote the right module category constructed from the pair $(H, \mu)$, where $H$ is a subgroup of $G$ such that $\left.\omega\right|_{H \times H \times H}$ is trivial in $H^{3}\left(H, k^{\times}\right)$ and $\mu \in C^{2}(G, C)$ is a 2-cochain satisfying $\delta^{2} \mu=\omega$. In the previous equation we regarded $\omega$ as an element of $Z^{3}(G, C)$ by treating $\omega\left(g_{1}, g_{2}, g_{3}\right)$ as a constant function on $K$, for all $g_{1}, g_{2}, g_{3} \in G$. The module category structure of $\mathcal{M}$ is given by $\mu$. If $\omega \equiv 1$, then we will assume that $\mu$ belongs to $Z^{2}\left(H, k^{\times}\right)$and that the module category structure of $\mathcal{M}(H, \mu)$ is given by $\varphi(\mu)$ (see (17) $)$. 
Definition 3.1. For each $y \in K^{H}$, define the set $\operatorname{Fun}_{y}=F u n_{y}(G, C)$ :

$$
\text { Fun }_{y}:=\left\{\gamma \in C^{1}(G, C) \mid \delta^{1} \gamma=\frac{{ }^{y} \mu}{\mu}\right\}
$$

Lemma 3.2. Invertible objects in $\mathcal{C}_{\mathcal{M}}^{*}$ are given by pairs $(\gamma, y)$, where $y \in K^{H}$ and $\gamma \in$ Fun .

Proof. We associate an invertible objects in $\mathcal{C}_{\mathcal{M}}^{*}$ to each pair $(\gamma, y)$, where $y \in K^{H}$ and $\gamma \in$ Fun $_{y}$ as follows: define a map $f_{y}: K \rightarrow K$ by $f_{y}(x)=p(u(y) u(x))$ for any $x \in K$. Extend the map $f_{y}$ to a functor $F_{y}: \mathcal{M} \rightarrow \mathcal{M}$. The module functor structure on $F_{y}$, which is also denoted by $\gamma$, is: $\gamma_{x, g}:=\gamma(g)(x) i d_{p(u(y) u(x \triangleleft g))}$ for any $g \in G$ and $x \in K$. The pentagon axiom for a module functor (13) is:

$$
{ }^{y} \mu\left(g_{1}, g_{2}\right)(x) \gamma\left(g_{1} g_{2}\right)(x)=\gamma\left(g_{1}\right)(x) \gamma\left(g_{2}\right)\left(x \triangleleft g_{1}\right) \mu\left(g_{1}, g_{2}\right)(x)
$$

for all $g_{1}, g_{2} \in G$ and $x \in K$.

This condition is satisfied because $\gamma \in F u n_{y}$. The inverse of $\left(\gamma, F_{y}\right)$ is the module functor associated to the pair $\left({ }^{p\left(u(y)^{-1}\right)} \gamma^{-1}, p\left(u(y)^{-1}\right)\right)$, where ${ }^{p\left(u(y)^{-1}\right)} \gamma^{-1}$ is defined as:

$p\left(u(y)^{-1}\right) \gamma^{-1}(g)(x):=\gamma(g)\left(p\left(u(y)^{-1} u(x)\right)^{-1}\right.$, for $g \in G$ and $y \in K$. It should be clear that all invertible objects in $\mathcal{C}_{\mathcal{M}}^{*}$ arise in this way and the lemma is proved.

Two invertible $\mathcal{C}$-module functors $\left(\gamma^{1}, y_{1}\right)$ and $\left(\gamma^{2}, y_{2}\right)$ are isomorphic in $\mathcal{C}_{\mathcal{M}}^{*}$ iff $y_{1}=y_{2}$ and there exists an element $\alpha \in C$ such that $\gamma^{1}(g)(x)=\frac{\alpha(x \triangleleft g)}{\alpha(x)} \gamma^{2}(g)(x)$ for all $g \in G$ and $x \in K$.

This motivates us to define an equivalence relation on the set $F u n_{y}$ : we define two elements $\gamma^{1}, \gamma^{2} \in$ Fun $_{y}$, to be equivalent if there exists an $\alpha \in C$ such that

$$
\gamma^{1}(g)(x)=\frac{\alpha(x \triangleleft g)}{\alpha(x)} \gamma^{2}(g)(x)
$$

for all $g \in G$ and $x \in K$.

Let $\overline{F u n}_{y}$ denote the set of equivalence classs of $F u n_{y}$ under the aforementioned equivalence relation.

Lemma 3.3. For each $y \in K^{H}$, if $\overline{F u n}_{y} \neq \emptyset$, then there is a bijection between the sets $\overline{F u n}_{y}$ and $H^{1}(G, C)$ and hence there is a bijection between the sets $\overline{F u n}_{y}$ and $\widehat{H}$.

Proof. Fix some $\eta_{y} \in F u n_{y}$. Then the maps $F u n_{y} \rightarrow Z^{1}(G, C): \beta \mapsto \frac{\beta}{\eta_{y}}$ and $Z^{1}(G, C) \rightarrow$ Fun $_{y}: \gamma \mapsto \eta_{y} \gamma$ are inverse to each other. These maps induce a bijection between the sets $\overline{F u n}_{y}$ and $H^{1}(G, C)$ The second statement of the lemma follows from Shapiro's Lemma.

Theorem 3.4. The tensor category $\mathcal{C}_{\mathcal{M}}^{*}$ (where $\mathcal{M}$ is the $\mathcal{C}=V(G, \omega)$-module category constructed from the pair $(H, \mu)$ where $H$ is a subgroup of $G$ such that $\left.\omega\right|_{H \times H \times H}$ is trivial in $H^{3}\left(H, k^{\times}\right)$and $\mu \in C^{2}(G, C)$ is a 2-cochain satisfying $\left.\delta^{2} \mu=\omega\right)$ is pointed if and only if the following three conditions hold:

(1) $H$ is abelian,

(2) $H$ is normal in $G$ and

(3) the restriction $\psi\left({ }^{y} \mu / \mu\right)$ is trivial in $H^{2}\left(H, k^{\times}\right)$, for all $y \in K$.

If $\omega \equiv 1$, then we assume that $\mu$ belongs to $Z^{2}\left(H, k^{\times}\right)$and the module category structure on $\mathcal{M}$ is given by $\varphi(\mu)$ (see (7)). The third condition above is then replaced with: 
$\left(3^{\prime}\right) \mu$ represents a $G$-invariant class in $H^{2}\left(H, k^{\times}\right)$.

Proof. Suppose that $\mathcal{C}_{\mathcal{M}}^{*}$ is pointed and let $\mathcal{S}=K^{H}$. The set of isomorphism classes of simple objects in $\mathcal{C}_{\mathcal{M}}^{*}$ is given by the set $\bigcup_{s \in \mathcal{S}}\left(\overline{F u n_{s}} \times\{s\}\right)$. By the previous Lemma, we have $F \operatorname{Pdim}\left(\mathcal{C}_{\mathcal{M}}^{*}\right) \leq|\widehat{H}||\mathcal{S}|$. Note that $|\widehat{H}| \leq|H|$ and $|\mathcal{S}| \leq|K|=\frac{|G|}{|H|}$. By Remark 2.10, $F P \operatorname{dim}\left(\mathcal{C}_{\mathcal{M}}^{*}\right)=F P \operatorname{dim}(\mathcal{C})=|G|$. It follows that we must have $\overline{F u n_{y}} \neq \emptyset$ for all $y \in K$, $|\widehat{H}|=|H|$ and $\mathcal{S}=K$. The second condition in the previous sentence means that $H$ is abelian. The third condition means that $H$ is normal in $G$. The first condition is equivalent to saying that $\frac{{ }^{y} \mu}{\mu}$ is trivial in $H^{2}(G, C)$, for all $y \in K$. This is equivalent to saying that the restriction $\psi\left(\frac{y}{\mu}\right)$ is trivial in $H^{2}\left(H, k^{\times}\right)$, for all $y \in K$.

Conversely, suppose that $H$ is abelian and normal in $G$ and that $\psi\left(\frac{y}{\mu}\right)$ is trivial in $H^{2}\left(H, k^{\times}\right)$, for all $y \in K$. Let $\mathcal{C}^{\prime}$ denote the full subcategory of $\mathcal{C}_{\mathcal{M}}^{*}$ of invertible objects. The isomorphism classes of invertible objects in the category $\mathcal{C}_{\mathcal{M}}^{*}$ are given by elements of the set $\bigcup_{x \in K}\left(\overline{F u n_{x}} \times\{x\}\right)$ and the size of each set in the previous union is $|H|$. So $F P \operatorname{dim}\left(\mathcal{C}^{\prime}\right)=|G|$. It follows that $\mathcal{C}_{\mathcal{M}}^{*}=\mathcal{C}^{\prime}$. In other words, every simple object in $\mathcal{C}_{\mathcal{M}}^{*}$ is invertible, that is, the category $\mathcal{C}_{\mathcal{M}}^{*}$ is pointed.

The last statement of the theorem follows from Lemma 2.3 .

Example 3.5. If $G=\mathbb{Z} / n \mathbb{Z}$ is a finite cyclic group, then $H^{2}\left(H, k^{\times}\right)=\{1\}$ for any subgroup $H$ of $G$. Hence the dual of $\operatorname{Vec}(G, \omega)$ with respect to any indecomposable module category for any 3-cocycle $\omega$ on $G$ is pointed. Also, for any abelian group $G$, the dual of $V e c(G)$ with respect to any indecomposable module category is pointed. On the other hand, the previous statement is not true for $\operatorname{Vec}(G, \omega)$ if $\omega$ is a non-trivial 3-cocycle on the abelian group $G$. Indeed, consider the dihedral group $D_{8}=\left\{r, s \mid r^{4}=s^{2}=1, r s=s r^{-1}\right\}$ and a subgroup $<r>$ of it. It can be shown that $\operatorname{Vec}\left(D_{8}\right)_{\mathcal{M}(<r>, 1)}^{*} \cong \operatorname{Vec}\left((\mathbb{Z} / 2 \mathbb{Z})^{3}, \omega\right)$, where $\omega$ is a non-trivial 3 -cocycle on $(\mathbb{Z} / 2 \mathbb{Z})^{3}$. Now, we know that $\operatorname{Vec}\left(D_{8}\right)$ is dual to the representation category $\operatorname{Rep}\left(D_{8}\right)$. Hence, there must exist an indecomposable module category over $\operatorname{Vec}\left((\mathbb{Z} / 2 \mathbb{Z})^{3}, \omega\right)$ with respect to which the dual of $\operatorname{Vec}\left((\mathbb{Z} / 2 \mathbb{Z})^{3}, \omega\right)$ is equivalent to the nonpointed tensor category $\operatorname{Rep}\left(D_{8}\right)$. We refer the reader to Coste, Gannon, and Ruelle, 2000 and Goff, Mason, and Ng, 2006 for similar results.

\section{The tensor CATEgory $\mathcal{C}_{\mathcal{M}}^{*}$ When IT IS POINTED}

In this section we further assume that $H$ is abelian and normal in $G$ and that $\frac{{ }^{y} \mu}{\mu}$ is trivial in $H^{2}(G, C)$, for all $y \in K$.

4.1. Tensor product and composition of morphisms in $\mathcal{C}_{\mathcal{M}}^{*}$. It suffices to restrict ourselves to simple objects in $\mathcal{C}_{\mathcal{M}}^{*}$. Recall that simple objects in $\mathcal{C}_{\mathcal{M}}^{*}$ are given by pairs $(\gamma, x)$, where $\gamma \in$ Fun $_{x}$ and $x \in K$. The element $x \in K$ determines a $\mathcal{C}$-module functor $F_{x}: \mathcal{M} \rightarrow \mathcal{M}$ given by $F_{x}(y)=x y$, for any $y \in K$. The $\mathcal{C}$-module functor structure on $F_{x}$ is given by $\gamma$. Tensor product (=composition of module functors) in $\mathcal{C}_{\mathcal{M}}^{*}$ : for any two simple objects $\left(\gamma^{1}, x_{1}\right)$ and $\left(\gamma^{2}, x_{2}\right),\left(\gamma^{1}, x_{1}\right) \otimes\left(\gamma^{2}, x_{2}\right)=\left({ }^{x_{2}} \gamma^{1} \gamma^{2}, x_{1} x_{2}\right)$ where ${ }^{x_{2}} \gamma^{1} \gamma^{2}$ is an element of the set Fun $x_{x_{1} x_{2}}$ and ${ }^{x_{2}} \gamma^{1}$ is defined as follows: ${ }^{x_{2}} \gamma^{1}(g)(y)=\gamma^{1}(g)\left(x_{2} y\right)$, for $g \in G, y \in K$.

Now let us look at morphisms in $\mathcal{C}_{\mathcal{M}}^{*}$. It suffices to restrict ourselves to isomorphisms between simple objects. Recall that an isomorphism between two simple objects $\left(\gamma^{1}, x\right)$ and $\left(\gamma^{2}, x\right)$ (note that the second coordinates have to be equal for an isomorphism to exist) in 
$\mathcal{C}_{\mathcal{M}}^{*}$ is given by an element $\alpha \in C$ which satisfies: $\gamma^{1}(g)(y)=\frac{\alpha(y \triangleleft g)}{\alpha(y)} \gamma^{2}(g)(y)$, for all $g \in G$ and $y \in K$.

Note 4.1. An isomorphism $\alpha:\left(\gamma^{1}, x\right) \rightarrow\left(\gamma^{2}, x\right)$ is completely determined by $\alpha(1)$. If $\alpha$ is an automorphism, then $\alpha(y)=\alpha(1)$ for all $y \in K$.

Now let us look at tensor product and composition of isomorphisms in $\mathcal{C}_{\mathcal{M}}^{*}$. Let $\alpha$ : $\left(\gamma^{1}, x_{1}\right) \rightarrow\left(\gamma^{2}, x_{1}\right)$ and $\beta:\left(\gamma^{3}, x_{2}\right) \rightarrow\left(\gamma^{4}, x_{2}\right)$ be any two isomorphisms between simple objects in $\mathcal{C}_{\mathcal{M}}^{*}$. The tensor product of $\alpha$ and $\beta$ : $(\alpha \otimes \beta)(x)=\left({ }^{x_{2}} \alpha \beta\right)(x)=\alpha\left(x_{2} x\right) \beta(x)$ for any $x \in K$. If $\gamma^{2}=\gamma^{3}$, then the composition of $\alpha$ and $\beta$ is given by $(\beta \circ \alpha)(x)=\beta(x) \alpha(x)$ for $x \in K$.

4.2. Grothendieck ring of the category $\mathcal{C}_{\mathcal{M}}^{*}$. The set of isomorphism classes of simple objects in $\mathcal{C}_{\mathcal{M}}^{*}$ form a group:

$$
\Lambda=\bigcup_{x \in K}\left(\overline{F u n}_{x} \times\{x\}\right) \quad\left(\overline{\gamma^{1}}, x_{1}\right) \star\left(\overline{\gamma^{2}}, x_{2}\right)=\left(\overline{x_{2} \gamma^{1} \gamma^{2}}, x_{1} x_{2}\right)
$$

where for any $\gamma \in$ Fun $_{x}$, by $\bar{\gamma}$ we mean the equivalence class of $\gamma$ in $\overline{F u n}_{x}$. The inverse of any $(\bar{\gamma}, x) \in \Lambda$ is $\left(\overline{\gamma_{x^{-1}}^{-1}}, x^{-1}\right)$. The Grothendieck ring $\mathcal{K}_{0}\left(\mathcal{C}_{\mathcal{M}}^{*}\right)$ equals $\mathbb{Z}[\Lambda]$.

The rest of the subsection is devoted to showing that $\Lambda$ is isomorphic to a certain crossed product of the groups $\widehat{H}$ and $K$.

Since we assumed that $\frac{y_{\mu} \mu}{\mu}$ is trivial in $H^{2}(G, C)$, for each $y \in K$ we have a map $\eta_{y} \in$ $C^{1}(G, C)$ such that:

$$
\delta^{1} \eta_{y}=\frac{{ }^{y} \mu}{\mu}
$$

Define a function

$$
\tilde{\nu}: K \times K \rightarrow C^{1}(G, C), \quad \tilde{\nu}\left(y_{1}, y_{2}\right)=\frac{{ }^{y_{2}} \eta_{y_{1}} \eta_{y_{2}}}{\eta_{y_{1} y_{2}}}
$$

Lemma 4.2. The function $\tilde{\nu}$ defines an element in $\underline{H}^{2}\left(K, H^{1}(G, C)\right)$.

Proof. Let us first show that $\tilde{\nu}\left(y_{1}, y_{2}\right) \in Z^{1}(G, C)$ for any $y_{1}, y_{2} \in K$. We have $\delta \eta_{y_{1} y_{2}}=$ $\frac{y_{1} y_{2} \mu}{\mu}=\frac{y_{2}\left(y_{1} \mu\right)}{\mu}=\frac{y_{2}\left(\delta \eta_{y_{1}} \mu\right)}{\mu}=\frac{y_{2}\left(\delta \eta_{y_{1}}\right) y_{2} \mu}{\mu}=\delta\left({ }^{y_{2}} \eta_{y_{1}}\right) \delta \eta_{y_{2}}=\delta\left({ }^{y_{2}} \eta_{y_{1}} \eta_{y_{2}}\right)$. So $\tilde{\nu}\left(y_{1}, y_{2}\right) \in$ $Z^{1}(G, C)$ for any $y_{1}, y_{2} \in K$. Now let us show that $\tilde{\nu} \in Z^{2}\left(K, Z^{1}(G, C)\right)$. We have

$$
\begin{aligned}
& (\delta \tilde{\nu})\left(y_{1}, y_{2}, y_{3}\right)=\tilde{\nu}\left(y_{2}, y_{3}\right) \tilde{\nu}\left(y_{1} y_{2}, y_{3}\right)^{-1} \tilde{\nu}\left(y_{1}, y_{2} y_{3}\right)\left(y_{3} \tilde{\nu}\left(y_{1}, y_{2}\right)\right)^{-1} \\
& =\frac{{ }^{y_{3}} \eta_{y_{2}} \eta_{y_{3}}}{\eta_{y_{2} y_{3}}} \times \frac{\eta_{y_{1} y_{2} y_{3}}}{{ }^{3} \eta_{y_{1} y_{2}} \eta_{y_{3}}} \times \frac{{ }_{y_{2} y_{3}} \eta_{y_{1}} \eta_{y_{2} y_{3}}}{\eta_{y_{1} y_{2} y_{3}}} \times \frac{{ }^{{ }_{3}} \eta_{y_{1} y_{2}}}{y_{3}\left(y_{2} \eta_{y_{1}}\right)^{y_{3}} \eta_{y_{2}}} \\
& \equiv 1
\end{aligned}
$$

The cohomology class of $\tilde{\nu}$ does not depend on the choice of the family of maps $\left\{\eta_{y} \mid y \in K\right\}$. Indeed, let $\left\{\eta_{y}^{\prime} \mid y \in K\right\}$ be another family of maps satisfying $\left(\delta \eta_{y}^{\prime}\right)=\frac{{ }^{y} \mu}{\mu}$ for all $y \in K$. We want to show that $\tilde{\nu}\left(y_{1}, y_{2}\right)=\frac{y_{2} \eta_{y_{1}} \eta_{y_{2}}}{\eta_{y_{1} y_{2}}}$ and $\tilde{\nu}^{\prime}\left(y_{1}, y_{2}\right)=\frac{y_{2} \eta_{y_{1}}^{\prime} \eta_{y_{2}}^{\prime}}{\eta_{y_{1} y_{2}}^{\prime}}$ define the same class in $\underline{H}^{2}\left(K, Z^{1}(G, C)\right)$. We have, $\delta\left(\frac{\eta_{y}}{\eta_{y}^{\prime}}\right)=1$, i.e. $\frac{\eta_{y}}{\eta_{y}^{\prime}} \in Z^{1}(G, C)$ for each $y \in K$. Define 
$\beta: K \rightarrow Z^{1}(G, C)$ by $\beta(y):=\frac{\eta_{y}}{\eta_{y}^{\prime}}$. Then, $\tilde{\nu}\left(y_{1}, y_{2}\right)=\frac{y_{2} \eta_{y_{1}} \eta_{y_{2}}}{\eta_{y_{1} y_{2}}}=\frac{y_{2} \beta\left(y_{1}\right) y_{2} \eta_{y_{1}}^{\prime} \beta\left(y_{2}\right) \eta_{y_{2}}^{\prime}}{\beta\left(y_{1} y_{2}\right) \eta_{y_{1} y_{2}}^{\prime}}=$ $(\delta \beta)\left(y_{1}, y_{2}\right) \tilde{\nu}^{\prime}\left(y_{1}, y_{2}\right)$.

Corollary 4.3. The function $\nu=\psi_{1} \circ \tilde{\nu}$ defines an element in $\underline{H}^{2}(K, \widehat{H})$.

Proof. The proof follows immediately from Lemmas 2.3 and 4.2 .

Remark 4.4. If $\omega \equiv 1$, then the element $\nu$ in the previous corollary is the image of $\mu$ under the following composition.

$$
\Phi: H^{2}\left(H, k^{\times}\right)^{K} \longrightarrow H^{2}(G, C)^{K} \longrightarrow \underline{H}^{2}\left(K, H^{1}(G, C)\right) \longrightarrow \underline{H}^{2}(K, \widehat{H})
$$

The first map in the above composition comes from $\varphi$ (17), the second from (19) and third is induced from the map $\psi_{1}$ (5). Maps similar to the one in (20) appears in Davydov, 2001 and Etingof and Gelaki, 2001.

Let us put a group structure on the set $\widehat{H} \times K$. For any two pairs $\left(\rho_{1}, x_{1}\right),\left(\rho_{2}, x_{2}\right)$ define their product by:

$$
\left(\rho_{1}, x_{1}\right)\left(\rho_{2}, x_{2}\right)=\left(\nu\left(x_{1}, x_{2}\right) \rho_{1}^{x_{2}} \rho_{2}, x_{1} x_{2}\right)
$$

Associativity follows from corollary 4.3. We denote this group by $\widehat{H} \rtimes_{\nu} K$. The group that we just constructed is known as a crossed product.

As mentioned in Lemma 3.3, the sets $\overline{F u n}_{x}$ and $\widehat{H}$ are in bijection for each $x \in K$. The following maps induce this bijection:

$$
\begin{aligned}
\zeta_{x}: \widehat{H} \rightarrow \text { Fun }_{x}, & \zeta_{x}(\rho):=\eta_{x} \varphi_{1}(\rho) \\
\theta_{x}: \text { Fun }_{x} \rightarrow \widehat{H}, & \theta_{x}(\gamma):=\psi_{1}\left(\gamma / \eta_{x}\right)
\end{aligned}
$$

where the maps $\varphi_{1}$ and $\psi_{1}$ were defined in (41) and (15), respectively.

Theorem 4.5. The Grothendieck ring $\mathcal{K}_{0}\left(\mathcal{C}_{\mathcal{M}}^{*}\right)=\mathbb{Z}[\Lambda]$ is isomorphic to the group ring $\mathbb{Z}\left[\widehat{H} \rtimes_{\nu} K\right]$.

Proof. Suffices to show that the groups $\Lambda$ and $\widehat{H} \rtimes_{\nu} K$ are isomorphic. Define a map $T$ : $\widehat{H} \rtimes_{\nu} K \rightarrow \Lambda$ by $T((\rho, x))=\left(\overline{\zeta_{x}(\rho)}, x\right)$. Let us show that $T$ is a group homomorphism. For any $\left(\rho_{1}, x_{1}\right),\left(\rho_{2}, x_{2}\right) \in \widehat{H} \rtimes_{\nu} K$, we have

$$
\begin{aligned}
T\left(\left(\rho_{1}, x_{1}\right)\left(\rho_{2}, x_{2}\right)\right) & =T\left(\left(\nu\left(x_{1}, x_{2}\right) \rho_{1}^{x_{2}} \rho_{2}, x_{1} x_{2}\right)\right) \\
& =\left(\overline{\zeta_{x_{1} x_{2}}\left(\nu\left(x_{1}, x_{2}\right) \rho_{1}^{x_{2}} \rho_{2}\right)}, x_{1} x_{2}\right)
\end{aligned}
$$

and,

$$
\begin{aligned}
& T\left(\left(\rho_{1}, x_{1}\right)\right) \star T\left(\left(\rho_{2}, x_{2}\right)\right)=\left(\overline{\zeta_{x_{1}}\left(\rho_{1}\right)}, x_{1}\right) \star\left(\overline{\zeta_{x_{2}}\left(\rho_{2}\right)}, x_{2}\right) \\
& \left.=\overline{\left(x_{2}\left(\zeta_{x_{1}}\left(\rho_{1}\right)\right) \zeta_{x_{2}}\left(\rho_{2}\right)\right.}, x_{1} x_{2}\right)
\end{aligned}
$$

Now, we show that $\theta_{x_{1} x_{2}}\left({ }^{x_{2}} \zeta_{x_{1}}\left(\rho_{1}\right) \zeta_{x_{2}}\left(\rho_{2}\right)\right)=\nu\left(x_{1}, x_{2}\right) \rho_{1}^{x_{2}} \rho_{2}$. For any $h \in H$, we have 


$$
\begin{aligned}
\theta_{x_{1} x_{2}}\left({ }^{x_{2}}\left(\zeta_{x_{1}}\left(\rho_{1}\right)\right) \zeta_{x_{2}}\left(\rho_{2}\right)\right)(h) & =\frac{\left({ }^{x_{2}}\left(\zeta_{x_{1}}\left(\rho_{1}\right)\right) \zeta_{x_{2}}\left(\rho_{2}\right)\right)(h)(1)}{\eta_{x_{1} x_{2}}(h)(1)} \\
& =\frac{\left(\zeta_{x_{1}}\left(\rho_{1}\right)(h)\right)\left(x_{2}\right)\left(\zeta_{x_{2}}\left(\rho_{2}\right)(h)\right)(1)}{\eta_{x_{1} x_{2}}(h)(1)} \\
& =\frac{\left(\varphi_{1}\left(\rho_{1}\right)(h)\right)\left(x_{2}\right) \eta_{x_{1}}(h)\left(x_{2}\right)\left(\varphi_{1}\left(\rho_{2}\right)(h)\right)(1) \eta_{x_{2}}(h)(1)}{\eta_{x_{1} x_{2}}(h)(1)} \\
& =\left(\nu\left(x_{1}, x_{2}\right) \rho_{1}^{x_{2}} \rho_{2}\right)(h)^{1}
\end{aligned}
$$

Hence, $\overline{\zeta_{x_{1} x_{2}}\left(\nu\left(x_{1}, x_{2}\right) \rho_{1}^{x_{2}} \rho_{2}\right)}=\overline{x_{2}\left(\zeta_{x_{1}}\left(\rho_{1}\right)\right) \zeta_{x_{2}}\left(\rho_{2}\right)}$. This shows that $\mathrm{T}$ is a homomorphism. It should be clear that $T$ is an isomorphism and the theorem is proved.

Example 4.6. Let $H$ be abelian and normal in $G$ such that its order is relatively prime to the order of the group $K$ and suppose $\psi\left({ }^{y} \mu / \mu\right)$ is trivial in $H^{2}\left(H, k^{\times}\right)$, for all $y \in K$. Then the Grothendieck ring of $\operatorname{Vec}(G, \omega)_{\mathcal{M}(H, \mu)}^{*}$ is $\mathbb{Z}[\widehat{H} \rtimes K]$. Indeed, since $|H|$ and $|K|$ are relatively prime we have $H^{2}(K, \widehat{H})=\{1\}$ which implies that $\nu$ is trivial in $H^{2}(K, \widehat{H})$.

4.3. Skeleton of the category $\mathcal{C}_{\mathcal{M}}^{*}$. A skeleton of a category $\mathcal{D}$ is any full subcategory $\overline{\mathcal{D}}$ such that each object of $\mathcal{D}$ is isomorphic (in $\mathcal{D}$ ) to exactly one object of $\overline{\mathcal{D}}$. Every category is equivalent to any of its skeletons. Let us recall how one constructs a skeleton $\overline{\mathcal{D}}$ of any tensor category $\mathcal{D}$ with associativity contraint $a$ and tensor product $\otimes$. The construction is as follows: choose one object from each isomorphism class of objects in $\mathcal{D}$. Let $\operatorname{obj}(\overline{\mathcal{D}})$ be the set of all objects choosen above. For any $X \in \operatorname{obj}(\mathcal{D})$, by $\bar{X}$ we mean the object in $\overline{\mathcal{D}}$ that represents the object $X$.

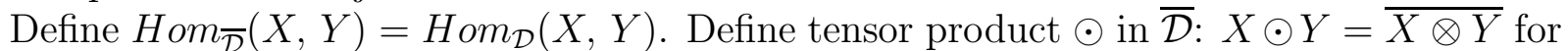
$X, Y \in \operatorname{Obj}(\overline{\mathcal{D}})$. Fix isomorphisms $\sigma(X, Y): X \odot Y \stackrel{\sim}{\rightarrow} X \otimes Y$ in $\mathcal{D}$, for all $X, Y \in \overline{\mathcal{D}}$. For any $f \in \operatorname{Hom}_{\overline{\mathcal{D}}}(X, Y)$ and $g \in \operatorname{Hom}_{\overline{\mathcal{D}}}\left(X^{\prime}, Y^{\prime}\right)$ define its tensor product: $f \odot g=\sigma\left(X^{\prime}, Y^{\prime}\right)^{-1} \circ$ $(f \otimes g) \circ \sigma(X, Y)$.

We now define associativity constraint $\bar{a}$ in $\overline{\mathcal{D}}$. For any $X, Y, Z \in \operatorname{obj}(\overline{\mathcal{D}})$ define $\bar{a}_{X, Y, Z}$ to be the following composition.

$$
\begin{gathered}
(X \odot Y) \odot Z \stackrel{\sigma(X \odot Y, Z)}{\longrightarrow}(X \odot Y) \otimes Z \stackrel{\sigma(X, Y) \otimes i d_{Z}}{\longrightarrow}(X \otimes Y) \otimes Z \stackrel{a_{X, Y, Z}}{\longrightarrow} X \otimes(Y \otimes Z) \\
\stackrel{\left(i d_{X} \otimes \sigma(Y, Z)\right)^{-1}}{\longrightarrow} X \otimes(Y \odot Z) \stackrel{\sigma(X, Y \odot Z)^{-1}}{\longrightarrow} X \odot(Y \odot Z)
\end{gathered}
$$

Left and right unit constraints are defined in the obvious way. It can be shown that the necessary axioms (pentagon, triangle) are satisfied. Hence $\overline{\mathcal{D}}$ is a monoidal category. One can also show that the categories $\mathcal{D}$ and $\overline{\mathcal{D}}$ are tensor equivalent.

Remark 4.7. If $\mathcal{D}$ is a pointed fusion category, then the simple objects of $\overline{\mathcal{D}}$ form a group and the associativity constraint in $\overline{\mathcal{D}}$ gives rise to a 3 -cocycle. The cohomology class of this 3 -cocycle does not depend on the choices made in the construction of $\overline{\mathcal{D}}$.

The function $\kappa$ defines an element in $Z^{2}(K, H)$ :

$$
\kappa\left(x_{1}, x_{2}\right):=\kappa_{x_{1}, u\left(x_{2}\right)} .
$$


Note that the cohomology class of $\kappa$ is independent on the choice of the function $u$. Also note that the cohomology class that $\kappa$ defines in $H^{2}(K, H)$ is equal to the cohomology class associated to the the exact sequence $1 \rightarrow H \rightarrow G \rightarrow K \rightarrow 1$.

Define a 3-cocycle on the group $\widehat{H} \rtimes_{\nu} K$ with coefficients in $k^{\times}$(see (21) and (19)):

$$
\varpi\left(\left(\rho_{1}, x_{1}\right),\left(\rho_{2}, x_{2}\right),\left(\rho_{3}, x_{3}\right)\right)=\left(\tilde{\nu}\left(x_{1}, x_{2}\right)\left(u\left(x_{3}\right)\right)\right)(1) \rho_{1}\left(\kappa\left(x_{2}, x_{3}\right)\right)
$$

for any $\left(\rho_{1}, x_{1}\right),\left(\rho_{2}, x_{2}\right),\left(\rho_{3}, x_{3}\right) \in \widehat{H} \rtimes_{\nu} K$.

Remark 4.8. (i) It is routine to check that $\varpi$ does indeed define a 3-cocycle and that its cohomology class does not depend on the choice of the function $u: K \rightarrow G$.

(ii) A special case, with $\tilde{\nu} \equiv 1$, of the formula in (24) appeared in Goff, Mason, and Ng, 2006.

Theorem 4.9. The fusion categories $\mathcal{C}_{\mathcal{M}}^{*}$ and $V e c\left(\widehat{H} \rtimes_{\nu} K, \varpi\right)$ are equivalent.

Proof. Let us construct a skeleton $\overline{\mathcal{C}_{\mathcal{M}}^{*}}$ of the category $\mathcal{C}_{\mathcal{M}}^{*}$. Let $\bar{\Lambda}=\bigcup_{x \in K}\left\{\left(\zeta_{x}(\rho), x\right) \mid \rho \in \widehat{H}\right\}$ denote the set of all simple objects of $\overline{\mathcal{C}_{\mathcal{M}}^{*}}$. See (22) for definition of $\zeta_{x}$. Tensor product $\odot$ in $\overline{\mathcal{C}_{\mathcal{M}}^{*}}:\left(\zeta_{x_{1}}\left(\rho_{1}\right), x_{1}\right) \odot\left(\zeta_{x_{2}}\left(\rho_{2}\right), x_{2}\right)=\overline{\left(\zeta_{x_{1}}\left(\rho_{1}\right), x_{1}\right) \otimes\left(\zeta_{x_{2}}\left(\rho_{2}\right), x_{2}\right)}=\overline{\left(x_{2}\left(\zeta_{x_{1}}\left(\rho_{1}\right)\right) \zeta_{x_{2}}\left(\rho_{2}\right), x_{1} x_{2}\right)}=$ $\left(\zeta_{x_{1} x_{2}}\left(\nu\left(x_{1}, x_{2}\right) \rho_{1}^{x_{2}} \rho_{2}\right), x_{1} x_{2}\right)$. Note that $\bar{\Lambda}$ forms a group (multiplication coming from $\odot$ ) that is isomorphic to $\widehat{H} \rtimes_{\nu} K$.

Fix isomorphisms in $\mathcal{C}_{\mathcal{M}}^{*}: C \ni f\left(\left(\zeta_{x_{1}}\left(\rho_{1}\right), x_{1}\right),\left(\zeta_{x_{2}}\left(\rho_{2}\right), x_{2}\right)\right):\left(\zeta_{x_{1}}\left(\rho_{1}\right), x_{1}\right) \odot\left(\zeta_{x_{2}}\left(\rho_{2}\right), x_{2}\right)=$ $\left(\zeta_{x_{1} x_{2}}\left(\nu\left(x_{1}, x_{2}\right) \rho_{1}^{x_{2}} \rho_{2}\right), x_{1} x_{2}\right) \stackrel{\sim}{\rightarrow}\left({ }^{x_{2}}\left(\zeta_{x_{1}}\left(\rho_{1}\right)\right) \zeta_{x_{2}}\left(\rho_{2}\right), x_{1} x_{2}\right)=\left(\zeta_{x_{1}}\left(\rho_{1}\right), x_{1}\right) \otimes\left(\zeta_{x_{2}}\left(\rho_{2}\right), x_{2}\right)$, for all $\left(\zeta_{x_{1}}\left(\rho_{1}\right), x_{1}\right),\left(\zeta_{x_{2}}\left(\rho_{2}\right), x_{2}\right) \in \bar{\Lambda}$. The following equality must hold:

$$
\begin{array}{r}
\left.x_{2}\left(\zeta_{x_{1}}\left(\rho_{1}\right)\right) \zeta_{x_{2}}\left(\rho_{2}\right)\right)(g)(y)=\frac{f\left(\left(\zeta_{x_{1}}\left(\rho_{1}\right), x_{1}\right),\left(\zeta_{x_{2}}\left(\rho_{2}\right), x_{2}\right)\right)(y \triangleleft g)}{f\left(\left(\zeta_{x_{1}}\left(\rho_{1}\right), x_{1}\right),\left(\zeta_{x_{2}}\left(\rho_{2}\right), x_{2}\right)\right)(y)} \\
\times \zeta_{x_{1} x_{2}}\left(\nu\left(x_{1}, x_{2}\right) \rho_{1}^{x_{2}} \rho_{2}\right)(g)(y)
\end{array}
$$

for all $g \in G, y \in K$. After using the definition of $\zeta_{x_{1}}, \zeta_{x_{2}}, \zeta_{x_{1} x_{2}}$ and $\tilde{\nu}$, canceling and rearranging, the above relation becomes:

$$
\frac{f\left(\left(\zeta_{x_{1}}\left(\rho_{1}\right), x_{1}\right),\left(\zeta_{x_{2}}\left(\rho_{2}\right), x_{2}\right)\right)(y \triangleleft g)}{f\left(\left(\zeta_{x_{1}}\left(\rho_{1}\right), x_{1}\right),\left(\zeta_{x_{2}}\left(\rho_{2}\right), x_{2}\right)\right)(y)}=\frac{\tilde{\nu}\left(x_{1}, x_{2}\right)(g)(y) \rho_{1}\left(\kappa_{x_{2} y, g}\right)}{\nu\left(x_{1}, x_{2}\right)\left(\kappa_{y, g}\right) \rho_{1}^{x_{2}}\left(\kappa_{y, g}\right)}
$$

Putting $y=1$ and $g=u(y)$ in the above relation and canceling, we obtain:

$$
f\left(\left(\zeta_{x_{1}}\left(\rho_{1}\right), x_{1}\right),\left(\zeta_{x_{2}}\left(\rho_{2}\right), x_{2}\right)\right)(y)=\frac{\tilde{\nu}\left(x_{1}, x_{2}\right)(u(y))(1) \rho_{1}\left(\kappa_{x_{2}, u(y)}\right)}{f\left(\left(\zeta_{x_{1}}\left(\rho_{1}\right), x_{1}\right),\left(\zeta_{x_{2}}\left(\rho_{2}\right), x_{2}\right)\right)(1)}
$$

Now let us calculate the associativity constraint in $\overline{\mathcal{C}_{\mathcal{M}}^{*}}$ which we denote by $\varpi^{\prime}$. For any $\left(\zeta_{x_{1}}\left(\rho_{1}\right), x_{1}\right),\left(\zeta_{x_{2}}\left(\rho_{2}\right), x_{2}\right),\left(\zeta_{x_{3}}\left(\rho_{3}\right), x_{3}\right) \in \bar{\Lambda}, \varpi^{\prime}$ is defined as:

$$
\varpi^{\prime}\left(\left(\zeta_{x_{1}}\left(\rho_{1}\right), x_{1}\right),\left(\zeta_{x_{2}}\left(\rho_{2}\right), x_{2}\right),\left(\zeta_{x_{3}}\left(\rho_{3}\right), x_{3}\right)\right)
$$




$$
\begin{aligned}
\left.\left.\left.=\frac{\left(f \left(\left(\zeta_{x_{1}}\left(\rho_{1}\right), x_{1}\right),\right.\right.}{f\left(\left(\zeta_{x_{1}}\left(\rho_{1}\right), x_{1}\right),\right.}\left(\zeta_{x_{2}}\left(\rho_{2}\right), x_{2}\right), x_{2}\right)\right) \otimes I d_{\left(\zeta_{x_{3}}\left(\rho_{3}\right), x_{3}\right)}\right) \\
\times \frac{\left.\left.f\left(\left(\zeta_{x_{3}}\left(\rho_{3}\right), x_{3}\right)\right), x_{1}\right) \odot\left(\zeta_{x_{2}}\left(\rho_{2}\right), x_{2}\right),\left(\zeta_{x_{3}}\left(\rho_{3}\right), x_{3}\right)\right)}{\left(I d_{\left(\zeta_{x_{1}}\left(\rho_{1}\right), x_{1}\right)} \otimes f\left(\left(\zeta_{x_{2}}\left(\rho_{2}\right), x_{2}\right),\left(\zeta_{x_{3}}\left(\rho_{3}\right), x_{3}\right)\right)\right)} \\
=\frac{{ }^{x_{3}}\left(f\left(\left(\zeta_{x_{1}}\left(\rho_{1}\right), x_{1}\right),\left(\zeta_{x_{2}}\left(\rho_{2}\right), x_{2}\right)\right)\right)}{f\left(\left(\zeta_{x_{1}}\left(\rho_{1}\right), x_{1}\right),\left(\zeta_{x_{2}}\left(\rho_{2}\right), x_{2}\right) \odot\left(\zeta_{x_{3}}\left(\rho_{3}\right), x_{3}\right)\right)} \\
\times \frac{f\left(\left(\zeta_{x_{1}}\left(\rho_{1}\right), x_{1}\right) \odot\left(\zeta_{x_{2}}\left(\rho_{2}\right), x_{2}\right),\left(\zeta_{x_{3}}\left(\rho_{3}\right), x_{3}\right)\right)}{f\left(\left(\zeta_{x_{2}}\left(\rho_{2}\right), x_{2}\right),\left(\zeta_{x_{3}}\left(\rho_{3}\right), x_{3}\right)\right)}
\end{aligned}
$$

Note that $\varpi^{\prime}\left(\left(\zeta_{x_{1}}\left(\rho_{1}\right), x_{1}\right),\left(\zeta_{x_{2}}\left(\rho_{2}\right), x_{2}\right),\left(\zeta_{x_{3}}\left(\rho_{3}\right), x_{3}\right)\right)$ is an automorphism of a simple object in $\mathcal{C}_{\mathcal{M}}^{*}$. By Note 4.1, $\varpi^{\prime}\left(\left(\zeta_{x_{1}}\left(\rho_{1}\right), x_{1}\right),\left(\zeta_{x_{2}}\left(\rho_{2}\right), x_{2}\right),\left(\zeta_{x_{3}}\left(\rho_{3}\right), x_{3}\right)\right)(y)$ is constant for all $y \in K$. Thus, it suffices to calculate $\varpi^{\prime}\left(\left(\zeta_{x_{1}}\left(\rho_{1}\right), x_{1}\right),\left(\zeta_{x_{2}}\left(\rho_{2}\right), x_{2}\right),\left(\zeta_{x_{3}}\left(\rho_{3}\right), x_{3}\right)\right)(1)$. We have,

$$
\begin{aligned}
\varpi^{\prime}\left(\left(\zeta_{x_{1}}\left(\rho_{1}\right), x_{1}\right),\left(\zeta_{x_{2}}\left(\rho_{2}\right), x_{2}\right),\left(\zeta_{x_{3}}\left(\rho_{3}\right), x_{3}\right)\right)(1) \\
=\frac{f\left(\left(\zeta_{x_{1}}\left(\rho_{1}\right), x_{1}\right),\left(\zeta_{x_{2}}\left(\rho_{2}\right), x_{2}\right)\right)\left(x_{3}\right)}{f\left(\left(\zeta_{x_{1}}\left(\rho_{1}\right), x_{1}\right),\left(\zeta_{x_{2}}\left(\rho_{2}\right), x_{2}\right) \odot\left(\zeta_{x_{3}}\left(\rho_{3}\right), x_{3}\right)\right)(1)} \\
\quad \times \frac{f\left(\left(\zeta_{x_{1}}\left(\rho_{1}\right), x_{1}\right) \odot\left(\zeta_{x_{2}}\left(\rho_{2}\right), x_{2}\right),\left(\zeta_{x_{3}}\left(\rho_{3}\right), x_{3}\right)\right)(1)}{f\left(\left(\zeta_{x_{2}}\left(\rho_{2}\right), x_{2}\right),\left(\zeta_{x_{3}}\left(\rho_{3}\right), x_{3}\right)\right)(1)} \\
=\frac{f\left(\left(\zeta_{x_{1}}\left(\rho_{1}\right), x_{1}\right),\left(\zeta_{x_{2}}\left(\rho_{2}\right), x_{2}\right)\right)(1)}{f\left(\left(\zeta_{x_{1}}\left(\rho_{1}\right), x_{1}\right),\left(\zeta_{x_{2}}\left(\rho_{2}\right), x_{2}\right) \odot\left(\zeta_{x_{3}}\left(\rho_{3}\right), x_{3}\right)\right)(1)} \\
\quad \times \frac{f\left(\left(\zeta_{x_{1}}\left(\rho_{1}\right), x_{1}\right) \odot\left(\zeta_{x_{2}}\left(\rho_{2}\right), x_{2}\right),\left(\zeta_{x_{3}}\left(\rho_{3}\right), x_{3}\right)\right)(1)}{\left.f\left(\left(\zeta_{x_{2}}\left(\rho_{2}\right), x_{2}\right),\left(\zeta_{x_{3}}\left(\rho_{3}\right)\right)\right), x_{3}\right)(1)} \\
\times \tilde{\nu}\left(x_{1}, x_{2}\right)\left(u\left(x_{3}\right)\right)(1) \rho_{1}\left(\kappa_{x_{2}, u\left(x_{3}\right)}\right)
\end{aligned}
$$

We used (25) to obtain the last equality.

Since the cohomology class of $\varpi^{\prime}$ does not depend on the choice of the isomorphisms $f(\cdot, \cdot)$, we can assume that $f(\cdot, \cdot)(1)=1$. Also, regard $\varpi^{\prime}$ as a 3 -cocycle on $\widehat{H} \rtimes_{\nu} K$. Then we get:

$$
\varpi^{\prime}\left(\left(\rho_{1}, x_{1}\right),\left(\rho_{2}, x_{2}\right),\left(\rho_{3}, x_{3}\right)\right)=\tilde{\nu}\left(x_{1}, x_{2}\right)\left(u\left(x_{3}\right)\right)(1) \rho_{1}\left(\kappa\left(x_{2}, x_{3}\right)\right)
$$

for any $\left(\rho_{1}, x_{1}\right),\left(\rho_{2}, x_{2}\right),\left(\rho_{3}, x_{3}\right) \in \widehat{H} \rtimes_{\nu} K$. That is, $\varpi^{\prime}=\varpi$ and the theorem is proved.

Example 4.10. Let $G=\mathbb{Z} / 4 \mathbb{Z}=\{\overline{0}, \overline{1}, \overline{2}, \overline{3}\}, \omega=1, H=\{\overline{0}, \overline{2}\}$, and $\mu \equiv 1$. Since $\mu \equiv 1$ we can assume that $\tilde{\nu} \equiv 1$ (see (19)) and $\nu \equiv 1$ (see Corollary 4.3). By Theorem 4.5] it follows that $\mathcal{K}_{0}\left(\mathcal{C}_{\mathcal{M}}^{*}\right) \cong \mathbb{Z}[\widehat{\mathbb{Z} / 2 \mathbb{Z}} \times \mathbb{Z} / 2 \mathbb{Z}]$. Let $\widehat{\mathbb{Z} / 2 \mathbb{Z}}=\left\{\rho_{0}, \rho_{1}\right\}$, where $\rho_{1}$ represents the nontrivial character. We have, $K=\{H+\overline{0}, H+\overline{1}\}$. We claim that the associativity constraint $\varpi$ in $\mathcal{C}_{\mathcal{M}}^{*}$ is non-trivial. It suffices to show that the restriction of $\varpi$ to some non-trivial subgroup of $\widehat{\mathbb{Z} / 2 \mathbb{Z}} \times \mathbb{Z} / 2 \mathbb{Z}$ is non-trivial. Consider the restriction of $\varpi$ to the subgroup $K=\left\{\left(\rho_{0}, H+\overline{0}\right),\left(\rho_{1}, H+\overline{1}\right)\right\}$. It suffices to show that there exists a triple of elements 
in this subgroup such that $\varpi$ evaluated at this triple is not equal to 1 . Define the function $u: K \rightarrow G$ by $u(H+\overline{0})=\overline{0}$ and $u(H+\overline{1})=\overline{1}$. Since $\mu \equiv 1$, we can choose $\tilde{\nu} \equiv 1$. So the first factor in the definition of $\varpi$ vanishes. We have, $\varpi\left(\left(\rho_{1}, H+\overline{1}\right),\left(\rho_{1}, H+\overline{1}\right),\left(\rho_{1}, H+\overline{1}\right)\right)=$ $\rho_{1}(\kappa(H+\overline{1}, H+\overline{1}))=\rho_{1}(\overline{2})=-1$. Thus, the 3-cocycle $\varpi$ is non-trivial. In particular, the fusion categories $\operatorname{Vec}(\mathbb{Z} / 4 \mathbb{Z}, 1)$ and $\operatorname{Vec}(\mathbb{Z} / 2 \mathbb{Z} \times \mathbb{Z} / 2 \mathbb{Z}, \varpi)$ are weakly Morita equivalent (see next section).

\section{Categorical Morita Equivalence}

Two tensor categories $\mathcal{C}$ and $\mathcal{D}$ are said to be weakly Morita equivalent if there exists an indecomposable right module category $\mathcal{M}$ over $\mathcal{C}$ such that the categories $\mathcal{C}_{\mathcal{M}}^{*}$ and $\mathcal{D}$ are tensor equivalent (see Müger, 2003). It was shown by Müger that this indeed is an equivalence relation.

Using the notion of weak Morita equivalence we put an equivalence relation on the set of all pairs $(G, \omega)$, where $G$ is a finite group and $\omega \in H^{3}\left(G, k^{\times}\right)$:

Definition 5.1. We say that two pairs $(G, \bar{\omega})$ and $\left(G^{\prime}, \bar{\omega}^{\prime}\right)$ are categorically Morita equivalent and write $(G, \bar{\omega}) \approx\left(G^{\prime}, \bar{\omega}^{\prime}\right)$ if the tensor categories $\operatorname{Vec}(G, \omega)$ and $\operatorname{Vec}\left(G^{\prime}, \omega^{\prime}\right)$, are weakly Morita equivalent.

Remark 5.2. Note that finding categorically Morita equivalence classes of the set of all pairs $(G, \omega)$, where $G$ is a finite group and $\omega \in H^{3}\left(G, k^{\times}\right)$amounts to finding weakly Morita equivalence classes of the set of all group-theoretical categories.

We also define an equivalence relation on the set of all groups:

Definition 5.3. We say that two groups $G$ and $G^{\prime}$ are categorically Morita equivalent and write $G \approx G^{\prime}$ if the pairs $(G, 1)$ and $\left(G^{\prime}, 1\right)$ are categorically Morita equivalent.

Remark 5.4. Two finite groups $G$ and $G^{\prime}$ are called isocategorical if their representation categories $\operatorname{Rep}(G)$ and $\operatorname{Rep}\left(G^{\prime}\right)$ are tensor equivalent Etingof and Gelaki, 2001. If two groups $G$ and $G^{\prime}$ are isocategorical, then they are categorically Morita equivalent (this follows from the fact that for any group $G$ the categories $\operatorname{Rep}(G)$ and $\operatorname{Vec}(G, 1)_{\mathcal{M}(G, 1)}^{*}$ are tensor equivalent). We show in Section 6 that the converse is not true, that is, there do exist groups that are categorically Morita equivalent but not isocategorical.

Remark 5.5. It was shown in Ostrik, $2003(2)$ that if the tensor categories $\mathcal{C}$ and $\mathcal{D}$ are weakly Morita equivalent, then their Drinfeld centers are equivalent as braided tensor categories. It follows that if two groups are categorically Morita equivalent, then the Drinfeld centers of their representation categories are equivalent as braided tensor categories.

Definition 5.6. We say that a group $G$ is categorically Morita rigid if any group that is categorically Morita equivalent to $G$ is actually isomorphic to $G$.

Remark 5.7. By remark 5.5 it follows that abelian groups are categorically Morita rigid. In particular, an abelian group can not be categorically Morita equivalent to a non-abelian group.

The next theorem gives a group-theoretical and cohomological interpretation of categorical Morita equivalence.

Theorem 5.8. Two pairs $(G, \bar{\omega})$ and $\left(G^{\prime}, \bar{\omega}^{\prime}\right)$ are categorically Morita equivalent if and only if the following conditions hold: 
(1) $G$ contains a normal abelian subgroup $H$ such that $\left.\omega\right|_{H \times H \times H}$ is trivial in $H^{3}\left(H, k^{\times}\right)$,

(2) there is a 2-cochain $\mu \in C^{2}(G, C)$ such that $\delta^{2} \mu=\omega$ and $\psi\left({ }^{y} \mu / \mu\right)$ is trivial in $H^{2}\left(H, k^{\times}\right)$, for all $y \in H \backslash G$ and there is an isomorphism $a: G^{\prime} \stackrel{\sim}{\rightarrow} \widehat{H} \rtimes_{\nu}(H \backslash G)$ (see (21)) and

(3) the 3-cocycle $\frac{\varpi^{a}}{\omega^{\prime}}$ is trivial in $H^{3}\left(G^{\prime}, k^{\times}\right)$(see (24) for definition of $\left.\varpi\right)$.

Proof. Suppose the pairs $(G, \bar{\omega})$ and $\left(G^{\prime}, \bar{\omega}^{\prime}\right)$ are categorically Morita equivalent. Then there exists an indecomposable right module category $\mathcal{M}$ over $\operatorname{Vec}(G, \omega)$ such that the categories $\operatorname{Vec}(G, \omega)_{\mathcal{M}}^{*}$ and $\operatorname{Vec}\left(G^{\prime}, \omega^{\prime}\right)$ are tensor equivalent. So there exists a subgroup $H$ of $G$ such that $\left.\omega\right|_{H \times H \times H}$ represents the trivial class in $H^{3}\left(H, K^{\times}\right)$and 2-cochain $\mu \in$ $C^{2}(G, C)$ (satisfying the $\delta^{2} \mu=\omega$ ) which together produce the module category $\mathcal{M}$. Note that $\operatorname{Vec}(G, \omega)_{\mathcal{M}}^{*}$ must be pointed. By Theorem 3.4, it follows that $H$ is abelian and normal in $G$ and that $\psi\left({ }^{y} \mu / \mu\right)$ is trivial in $H^{2}\left(H, k^{\times}\right)$, for all $y \in H \backslash G$. Theorem 4.9 says that $\operatorname{Vec}(G, \omega)_{\mathcal{M}}^{*} \cong \operatorname{Vec}\left(\widehat{H} \rtimes_{\nu}(H \backslash G), \varpi\right)$. It now follows that there must exist an isomorphism $a: G^{\prime} \rightarrow \widehat{H} \rtimes_{\nu}(H \backslash G)$ such that $\varpi^{a}$ is cohomologous to $\omega^{\prime}$. The converse should be clear and the theorem is proved.

Corollary 5.9. Two groups $G$ and $G^{\prime}$ are categorically Morita equivalent if and only if the following conditions hold:

(1) $G$ contains a normal abelian subgroup $H$,

(2) there exists a $G$-invariant $\mu \in H^{2}\left(H, k^{\times}\right)$such that the groups $G^{\prime}$ and $\widehat{H} \rtimes_{\nu}(H \backslash G)$ are isomorphic (where $\nu=\Phi(\mu)$, see (20)) and

(3) the 3-cocycle $\varpi$ defined in (24) is trivial.

\section{Examples of CATEgorically Morita Equivalent groups With NON-ISOMORPHIC GROTHENDIECK RINGS}

In this section we produce a series of pairs of groups that are categorically Morita equivalent but have non-isomorphic Grothendieck rings. Let $p$ and $q$ be odd primes such that $p-1$ is divisible by $q$. Then there exists a unique upto isomorphism non-trivial semidirect product of the groups $\mathbb{Z} / p \mathbb{Z}$ and $\mathbb{Z} / q \mathbb{Z}$. Let $a$ and $b$ be generators of the groups $\mathbb{Z} / p \mathbb{Z}$ and $\mathbb{Z} / q \mathbb{Z}$, respectively. Let us fix an action of $\mathbb{Z} / q \mathbb{Z}$ on $\mathbb{Z} / p \mathbb{Z}$ : fix a $t \in \mathbb{Z}(t \not \equiv 1 \bmod p)$ such that $t^{q}-1$ is divisible by $p$. Such a $t$ of course exists because $p-1$ is divisible by $q$. Then the action of $\mathbb{Z} / q \mathbb{Z}$ on $\mathbb{Z} / p \mathbb{Z}$ is defined by: $a \triangleleft b:=a^{t}$. Let $\rho$ be a generator of the groups $\widehat{\mathbb{Z} / p \mathbb{Z}}$. Then the induced action of $\mathbb{Z} / q \mathbb{Z}$ on $\widehat{\mathbb{Z} / p \mathbb{Z}}$ is given by: $(\rho \triangleleft b)(a):=\rho\left(a \triangleleft b^{-1}\right)$. But $b^{-1}=b^{q-1}$. So $\rho \triangleleft b=\rho^{t^{q-1}}$.

The subgroup $\mathbb{Z} / p \mathbb{Z}$ (identified with $\mathbb{Z} / p \mathbb{Z} \times\{1\}$ ) of $\mathbb{Z} / p \mathbb{Z} \rtimes \mathbb{Z} / q \mathbb{Z}$ can be considered as a right $(\mathbb{Z} / p \mathbb{Z} \rtimes \mathbb{Z} / q \mathbb{Z})$-module where the action is via conjugation. The dual group $\widehat{\mathbb{Z} / p \mathbb{Z}}$ is also a right $(\mathbb{Z} / p \mathbb{Z} \rtimes \mathbb{Z} / q \mathbb{Z})$-module with the action being induced from the action of

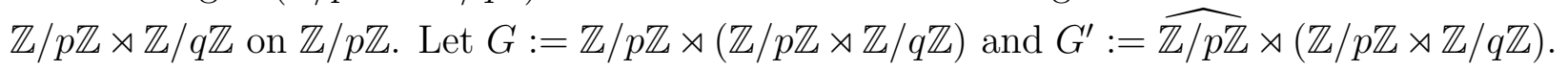

Lemma 6.1. The groups $G$ and $G^{\prime}$ have different number of normal subgroups of order $p$.

Proof. Note that both groups have the same number of subgroups of order $p$. We claim that all subgroups of order $p$ in $G$ are normal whereas there exists a non-normal subgroup of order $p$ in $G^{\prime}$. The generator of any subgroup of $G$ of order $p$ is of the form $\left(a^{l},\left(a^{m}, 1\right)\right)$, where $l, m \in\{1, \ldots, p\}$ with $l$ and $m$ not simultaneously equal to $p$. The elements $(a,(1,1))$, $(1,(a, 1))$, and $(1,(1, b))$ generate the group $G$. Note that the element $\left(a^{l},\left(a^{m}, 1\right)\right)$ is stable 
under conjugation by the first two generators of $G$. While conjugation by the third generator gives: $(1,(1, b))^{-1}\left(a^{l},\left(a^{m}, 1\right)\right)(1,(1, b))=\left(1,\left(1, b^{-1}\right)\right)\left(a^{l t},\left(a^{m t}, b\right)\right)=\left(a^{l t},\left(a^{m t}, 1\right)\right)=$ $\left(a^{l},\left(a^{m}, 1\right)\right)^{t}$. This shows that all subgroups of order $p$ in $G$ are normal. Consider the subgroup of $G^{\prime}$ of order $p$ generated by the element $(\rho,(a, 1))$. We have

$(1,(1, b))^{-1}(\rho,(a, 1))(1,(1, b))=\left(1,\left(1, b^{-1}\right)\right)\left(\rho^{t^{q-1}},\left(a^{t}, b\right)\right)=\left(\rho^{t^{q-1}},\left(a^{t}, 1\right)\right)$. Note that the element $\left(\rho^{t^{q-1}},\left(a^{t}, 1\right)\right)$ is not a power of $(\rho,(a, 1))$ because $t^{q-1} \not \equiv t \bmod p$. This shows that the subgroup of $G^{\prime}$ of order $p$ generated by the element $(\rho,(a, 1))$ is not normal and hence the lemma is proved.

Corollary 6.2. The groups $G$ and $G^{\prime}$ are categorically Morita equivalent but have nonisomorphic Grothendieck rings.

Proof. To see that these two groups satisfy the conditions in Corollary [5.9, take $H$ to be the subgroup $\mathbb{Z} / p \mathbb{Z}$ of $G$ and take $\mu \equiv 1$. Observe that the groups $\widehat{H} \rtimes(H \backslash G)$ and $G^{\prime}$ are isomorphic. Since the exact sequence $1 \rightarrow H \rightarrow G \rightarrow H \backslash G \rightarrow 1$ splits we can assume $\kappa \equiv 1$. Also, since $\mu \equiv 1$ we can assume that $\tilde{\nu} \equiv 1$ and therefore $\varpi \equiv 1$. It follows that the groups $G$ and $G^{\prime}$ are categorically Morita equivalent. To see that these groups have non-isomorphic Grothendieck rings note that the above lemma implies that these groups have different number of quotient groups of order $p q$. By Nikshych, 1998, Proposition 3.11] it follows that the Grothendieck rings $K_{0}(\operatorname{Rep}(G))$ and $K_{0}\left(\operatorname{Rep}\left(G^{\prime}\right)\right)$ of the two groups are not isomorphic.

Corollary 6.3. The groups $G$ and $G^{\prime}$ are non-isocategorical.

Proof. This follows immediately from the above corollary.

Remark 6.4. (i) By Remark 5.5 the representation categories $\operatorname{Rep}(D(G))$ and $\operatorname{Rep}\left(D\left(G^{\prime}\right)\right)$ of the Drinfeld doubles of the groups $G$ and $G^{\prime}$ are equivalent as braided tensor categories and hence these groups define the same modular data. Note also that the Hopf algebras $D(G)$ and $D\left(G^{\prime}\right)$ must be guage-equivalent.

(ii) Equivalence of certain twisted doubles of groups was investigated in Goff, Mason, and Ng, 2006.

(iii) The above examples of categorically Morita equivalent groups come from a more general construction: start with any finite group $G$ and a finite right $G$-module $H$. Consider the semidirect product $H \rtimes G$. We can regard $\widehat{H}$ as a right $G$-module with the action being induced from the action of $G$ on $H$. Then the groups $H \rtimes G$ and $\widehat{H} \rtimes G$ are categorically Morita equivalent. Note however that these two groups are not always non-isomorphic.

(iv) By Ito's theorem Grove, 1997, Theorem 6.3.9] it follows that the possible dimensions of irreducible representations of the groups $G$ and $G^{\prime}$ are 1 and $q$. It can be shown that the order of the commutator subgroup is $p^{2}$ for both groups. Therefore, the order of the abelianization (equal to the number of 1-dimensional representations) of both groups is $q$. So the group algebras $k G$ and $k G^{\prime}$ are both isomorphic to $\underbrace{k \oplus k \oplus \cdots \oplus k}_{q \text { copies }} \oplus \underbrace{M_{q}(k) \oplus M_{q}(k) \oplus \cdots \oplus M_{q}(k)}_{\left(p^{2}-1\right) / q \text { copies }}$.

(v) It follows from Corollary 6.2 that the groups $G$ and $G^{\prime}$ have different character tables. This provides a counter-example to the hunch mentioned in Coste, Gannon, and Ruelle, 2000 that groups defining the same modular data will have the same character table. 


\section{ACKNOWLEDGMENTS}

The author would like to express his deep gratitude to his dissertation advisor, Prof. Dmitri Nikshych, for introducing this problem to him and for providing invaluable help and guidance during the preparation of this paper. The author would also like to thank the referee for comments that helped improve the paper. Thanks are also due to Shamindra Ghosh for useful discussions. During preparation of the paper the author was supported by fellowships awarded by the graduate school at the University of New Hampshire and by NSF grant DMS-0200202.

\section{REFERENCES}

[Bakalov and Kirillov Jr., 2001] B. Bakalov, A. Kirillov Jr., Lectures on tensor categories and modular functors, University Lecture Series, vol. 21, American Mathematical Society, Rhode Island, 2001.

[Coste, Gannon, and Ruelle, 2000] A. Coste, T. Gannon, and P. Ruelle, Finite group modular data, Nuclear Phys. B 581 (2000), no. 3, 679-717.

[Davydov, 2001] A. A. Davydov, Galois Algebras and monoidal functors between categories of representations of finite groups, J. Algebra 244 (2001), 273-301.

[Dijkgraaf, Pasquier, and Roche, 1990] R. Dijkgraaf, V. Pasquier, and P. Roche, Quasi-quantum groups related to orbifold models, Nuclear Phys. B. Proc. Suppl. 18B (1990), 60-72.

[Etingof and Gelaki, 2001] P. Etingof and S. Gelaki, Isocategorical Groups, Int. Math. Res. Not., 2001, no. $2,59-76$.

[Etingof, Nikshych and Ostrik, 2005] P. Etingof, D. Nikshych, and V. Ostrik, On fusion categories, Ann. of Math. 162 (2005), 581-642.

[Etingof and Ostrik, 2003] P. Etingof and V. Ostrik, Finite tensor categories, Mosc. Math. J., 2003, no. 3, 627-654, 782-783.

[Karpilovsky, 1987] G. Karpilovsky, The Schur multiplier, London mathematical society monographs new series 2, Oxford science publications, Northern Ireland, 1987.

[Kassel, 1995] C. Kassel, Quantum groups, Graduate texts in mathematics 155, Springer-Verlag, New York, 1995.

[Goff, Mason, and Ng, 2006] C. Goff, G. Mason, and S. Ng, On the Gauge Equivalence of Twisted Quantum Doubles of Elementary Abelian and Extra-Special 2-Groups, 2006, http://www.arxiv.org/abs/math.QA/0603191

[Grove, 1997] L. C. Grove, Groups and Characters, Pure and applied mathematics, A Wiley-Interscience series of texts, monographs, and tracts, John Wiley \& sons, Inc., 1997.

[MacLane, 1998] S. MacLane, Categories for the working mathematician, Graduate texts in mathematics 5, 2nd edition, Springer-Verlag, 1998.

[Müger, 2003] M. Müger, From subfactors to categories and topology I. Frobenius algebras in and Morita equivalence of tensor categories, J. Pure Appl. Algebra 180 (2003), 81-157.

[Nikshych, 1998] D. Nikshych, $K_{0}$-rings and twisting of finite dimensional semisimple Hopf algebras, Commun. Alg. 26 (1998), 321-342.

[Ostrik, 2003 (1)] V. Ostrik, Module categories, weak Hopf algebras and modular invariants, Transform. Groups 8 (2003), no.2, 177-206.

[Ostrik, 2003 (2)] V. Ostrik, Module categories over the Drinfeld double of a finite group, Int. Math. Res. Not., 2003, no. 27, 1507-1520.

[Rotman, 1979] J. Rotman, An introduction to homological algebra, Academic Press, New York, 1979.

Department of Mathematics and Statistics, University of New Hampshire, Durham, NH 03824, USA

E-mail address: dnaidu@unh.edu 